\title{
Nonlinear integral equations for the thermodynamics of the sl(4)-symmetric Uimin-Sutherland model
}

\author{
Jens Damerau* and Andreas Klümper ${ }^{\dagger}$ \\ Fachbereich C - Physik, Bergische Universität Wuppertal, \\ 42097 Wuppertal, Germany
}

19th October 2006

\begin{abstract}
We derive a finite set of nonlinear integral equations (NLIE) for the thermodynamics of the one-dimensional sl(4)-symmetric Uimin-Sutherland model. Our NLIE can be evaluated numerically for arbitrary finite temperature and chemical potentials. We recover the NLIE for $s l(3)$ as a limiting case. In comparison to other recently derived NLIE, the evaluation at low temperature poses no problem in our formulation. The model shows a rich ground-state phase diagram. We obtain the critical fields from the $T \rightarrow 0$ limit of our NLIE. As an example for the application of the NLIE, we give numerical results for the $S U(4)$ spin-orbital model. The magnetic susceptibility shows divergences at critical fields in the low-temperature limit and logarithmic singularities for zero magnetic field.
\end{abstract}

PACS: 02.30.Ik, 05.70.-a, 75.10.Jm

\section{Introduction}

Since Bethe's seminal solution of the one-dimensional spin-1/2 Heisenberg chain [1], many integrable, natural generalisations of this model have been treated using basically the same ansatz. Among them is a multi-component, higher-rank generalisation of the Heisenberg chain, first proposed by Uimin for the case of three components [2]. Later, Sutherland introduced and solved the model for an arbitrary number of particle types [3]. The two-dimensional classical model associated with the one-dimensional Uimin-Sutherland (US) model is the Perk-Schultz (PS) model [4]. The case of higher-rank representations of the underlying symmetry algebra was treated by Andrei and Johannesson [5, 6]. Affleck calculated the critical behaviour based on non-Abelian bosonisation and conformal field theory [7, 8].

*e-mail: damerau@physik.uni-wuppertal.de

${ }^{\dagger}$ e-mail: kluemper@physik.uni-wuppertal.de 
The traditional thermodynamic Bethe ansatz (TBA) allows for the treatment of finite-temperature properties [9, 10, 11, 12]. It uses the string hypothesis and typically yields an infinite set of nonlinear integral equations (NLIE). The numerical solution of these equations poses a problem as some kind of truncation scheme is necessary. Only in the limit $T \rightarrow 0$ one obtains a finite set of equations. Using the TBA approach for the general US model [13], the low-field asymptotics of the susceptibility [14] as well as the low-temperature asymptotics of the specific heat [15, 16] have been derived analytically.

With the help of the quantum transfer matrix (QTM) formalism [17, 18] and the fusion hierarchy of transfer matrices [19, 20, 21, 22, 23, 24], it is possible to rederive the TBA equations without using the string hypothesis [25]. Using the QTM it is also possible to derive an alternative set of NLIE consisting only of a finite number of unknown auxiliary functions [26, 27, 28, 29], thus allowing for a precise numerical treatment at arbitrary finite temperature. Later it was realised that these auxiliary functions provide a natural way to exactly truncate the TBA equations [30]. Nevertheless, no straightforward way of getting the required auxiliary functions is known. Up to now, NLIE of this type have only been derived for three components at most [31, 32, 33].

There exists yet another type of NLIE [34], which allows for the generalisation to an arbitrary number of components [35]. Unfortunately, these NLIE prove difficult to evaluate at low temperature. Instead, high-temperature expansions (HTE) have been obtained up to high order [35, 36]. Very recently, these equations have been further generalised to treat $U_{q}(\widehat{s l}(r \mid s))$-symmetric PS models [37].

In this paper, we treat the four-state, sl(4)-symmetric US model in the spirit of [29]. We define 14 suitable auxiliary functions, from which we derive a set of well-posed NLIE that are valid for arbitrary finite temperature and chemical potentials. The auxiliary functions are connected to the fundamental representations of $s l(4)$. Our NLIE are the natural generalisation of those obtained for the $s l(3)$-symmetric case [33].

The $s l(4)$-symmetric US model has many interesting applications. As an example, we treat the $S U(4)$ spin-orbital model [38, 39]. The thermodynamic properties of this model have already been studied numerically using various methods [37, 40, 41, 42]. The ground-state phase diagram in dependence of the magnetic field and the orbital Landé factor has also been obtained [43]. In comparison to these methods, we are able to obtain highly accurate numerical results for low finite temperatures in the thermodynamic limit. Other possible applications of the $s l(4)$-symmetric US model include an integrable twoleg spin ladder system [44], which has recently been studied numerically using the HTE and TBA methods [45, 46].

The outline of this paper is as follows. In Section 2, we briefly introduce the $q$-state US model and show how its thermodynamic properties can be obtained using the QTM approach. In Section 3, we concentrate on the $\operatorname{sl}(4)$-symmetric case. We present a set of 14 well-posed auxiliary functions, from which we derive a closed set of NLIE. We show how the largest eigenvalue of the QTM can be extracted from these auxiliary functions. In Section 4, we treat two limiting cases of our NLIE. First, the NLIE and auxiliary functions of the $s l(3)$-symmetric case are recovered by freezing out one of the states. Second, the limit $T \rightarrow 0$ yields linearised integral equations, which are equivalent to the corresponding TBA equations and allow for the derivation of the critical fields. 


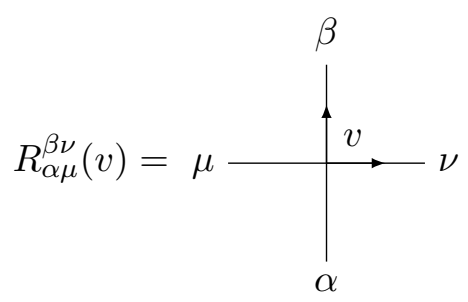

Figure 1: Graphical depiction of the $R$-matrix as defined in equation (3).

In Section 5, we deal with the numerical solution of our NLIE. We briefly introduce the $S U(4)$ spin-orbital model as an application and give results for various physical quantities. In Section 6, we give a summary of our work and an outlook on open problems. Appendix $\mathrm{A}$ is devoted to details concerning the derivation of the NLIE.

\section{QTM approach to the US model}

In order to fix notation, we begin with a short review of the Uimin-Sutherland (US) model [2, 3]. Consider a one-dimensional lattice with $L$ sites, where a $q$-state spin variable $\alpha_{j}$ is assigned to each site $j$. Each spin $\alpha$ has its own grading $\epsilon_{\alpha}=(-1)^{p(\alpha)}= \pm 1$. The Hamiltonian of the US model is then given by

$$
\mathcal{H}_{0}=\sum_{j=1}^{L} \pi_{j, j+1},
$$

where the local interaction operator $\pi_{j, j+1}$ permutes neighbouring spins on the lattice with respect to their grading,

$$
\pi_{j, j+1}\left|\alpha_{1} \ldots \alpha_{j} \alpha_{j+1} \ldots \alpha_{L}\right\rangle=(-1)^{p\left(\alpha_{j}\right) p\left(\alpha_{j+1}\right)}\left|\alpha_{1} \ldots \alpha_{j+1} \alpha_{j} \ldots \alpha_{L}\right\rangle,
$$

and periodic boundary conditions are imposed. The model shows $s l(r \mid s)$ symmetry, where $r$ and $s$ are the total number of states with positive and negative grading $(q=r+s)$, respectively. It is therefore a higher rank generalisation of the spin- $1 / 2$ Heisenberg chain, which is contained as the special case $q=2$ and $\epsilon_{1}=\epsilon_{2}=+1$.

The one-dimensional US model is known to be exactly solvable by Bethe ansatz (BA). The two-dimensional classical counterpart is given by the Perk-Schultz (PS) model [4], which is defined on a square lattice with $L \times N$ sites and periodic boundary conditions in both directions. Variables taking on integer values from 1 to $q$ are assigned to each bond of the lattice, and a Boltzmann weight depending on a spectral parameter $v$,

$$
R_{\alpha \mu}^{\beta \nu}(v)=\delta_{\alpha \nu} \delta_{\mu \beta}+v \cdot(-1)^{p(\alpha) p(\mu)} \cdot \delta_{\alpha \beta} \delta_{\mu \nu},
$$

is associated to every local vertex configuration $(\alpha, \beta, \mu, \nu)$, see Figure 1. We define the row-to-row transfer matrix

$$
\mathcal{T}_{\alpha}^{\beta}(v)=\sum_{\{\nu\}} \prod_{j=1}^{L} R_{\alpha_{j} \nu_{j}}^{\beta_{j} \nu_{j+1}}(v) .
$$


As the $R$-matrix (3) is a solution to the Yang-Baxter equation, these transfer matrices form a commuting family $\left[\mathcal{T}(v), \mathcal{T}\left(v^{\prime}\right)\right]=0$ for all $v, v^{\prime} \in \mathbb{C}$. Making use of Baxter's formula [47], one recovers the Hamiltonian of the US model from the transfer matrix of the PS model at the shift point $v=0$,

$$
\mathcal{H}_{0}=\left.\frac{\mathrm{d}}{\mathrm{d} v} \ln \mathcal{T}(v)\right|_{v=0}=\sum_{j=1}^{L} \pi_{j, j+1} .
$$

Without breaking integrability, we may add external field terms,

$$
\mathcal{H}=\mathcal{H}_{0}+\mathcal{H}_{\text {ext }}=\mathcal{H}_{0}-\sum_{j=1}^{L} \sum_{\alpha=1}^{q} \mu_{\alpha} n_{j, \alpha},
$$

where $\mu_{\alpha}$ is some general chemical potential associated with state $\alpha$ and the operator $n_{j, \alpha}$ counts the number of particles of type $\alpha$ sitting on site $j$.

We are interested in the thermodynamics of the US model. Hence, we want to establish some connection between its partition function and the transfer matrix of the PS model. We therefore consider a second $R$-matrix, namely $\bar{R}(v)$, obtained by rotating the graphical depiction of $R(v)$ clockwise by 90 degrees,

$$
\bar{R}_{\alpha \mu}^{\beta \nu}(v)=R_{\nu \alpha}^{\mu \beta}(v)
$$

We define the transfer matrix $\overline{\mathcal{T}}(v)$ as the product of matrices $\bar{R}(v)$ in analogy to (4). Equation (15) now also applies to $\overline{\mathcal{T}}(v)$, and as a consequence the relation

$$
\mathcal{T}(-\beta / N) \overline{\mathcal{T}}(-\beta / N)=\mathrm{e}^{-2(\beta / N) \mathcal{H}_{0}+\mathcal{O}\left((\beta / N)^{2}\right)}
$$

is valid for arbitrary inverse temperature $\beta$ and a sufficiently large even integer Trotter number $N$. The partition function of the one-dimensional US model is then given by

$$
Z=\operatorname{Tr} \mathrm{e}^{-\beta \mathcal{H}}=\lim _{N \rightarrow \infty} \operatorname{Tr}\left[(\mathcal{T}(u) \overline{\mathcal{T}}(u))^{N / 2} \mathrm{e}^{-\beta \mathcal{H} \text { ext }}\right],
$$

where $u=-\beta / N$ and the traces are taken in the $q^{L}$-dimensional space. Obviously, the partition function of the one-dimensional US model is equal to the partition function of a staggered two-dimensional PS model, where the external field can be incorporated by modifying the boundary conditions in the Trotter direction. Equation (9) is still difficult to evaluate, e.g. all eigenstates have to be taken into account. To avoid this problem, it is better to consider the column-to-column transfer matrix of the staggered vertex model, which is called the quantum transfer matrix (QTM) [17, 18]. In order to write down the QTM in a convenient way, we first define the matrix $\widetilde{R}(v)$, which we get by rotating $R(v)$ counterclockwise by 90 degrees and changing the sign of the spectral parameter,

$$
\widetilde{R}_{\alpha \mu}^{\beta \nu}(v)=R_{\mu \beta}^{\nu \alpha}(-v)
$$


Then the QTM takes the form

$$
\left(\mathcal{T}^{\mathrm{QTM}}\right)_{\alpha}^{\beta}(v)=\sum_{\{\nu\}} \mathrm{e}^{\beta \mu_{\nu_{1}}} \prod_{j=1}^{N / 2} R_{\alpha_{2 j-1} \nu_{2 j-1}}^{\beta_{2 j-1} \nu_{2 j}}(\mathrm{i} v+u) \widetilde{R}_{\alpha_{2 j} \nu_{2 j}}^{\beta_{2 j} \nu_{2 j+1}}(\mathrm{i} v-u),
$$

where we have introduced a new spectral parameter $v$, so that the QTMs for all $v, v^{\prime} \in \mathbb{C}$ form a commuting family,

$$
\left[\mathcal{T}^{\mathrm{QTM}}(v), \mathcal{T}^{\mathrm{QTM}}\left(v^{\prime}\right)\right]=0 .
$$

This allows for the diagonalisation by use of the BA. In the end, we are only interested in the case $v=0$ as the partition function of the one-dimensional US model in terms of the QTM is

$$
Z=\lim _{N \rightarrow \infty} \operatorname{Tr}\left(\mathcal{T}^{\mathrm{QTM}}(0)\right)^{L} .
$$

In the thermodynamic limit $(L \rightarrow \infty)$ one finds that the thermodynamics of the US model solely depends on the unique largest eigenvalue of the QTM [48, 49]. For the free energy per unit length, we finally get

$$
f=-\lim _{L \rightarrow \infty} \frac{1}{L \beta} \ln Z=-\frac{1}{\beta} \ln \Lambda_{\max }(0),
$$

where $\Lambda_{\max }(v)$ is the largest eigenvalue of the QTM. is 50 ]

As noted before, the QTM can be diagonalised via BA. The result for the eigenvalue

$$
\Lambda(v)=\sum_{j=1}^{q} \lambda_{j}(v)
$$

where

$$
\lambda_{j}(v)=\phi_{-}(v) \phi_{+}(v) \frac{q_{j-1}\left(v-\mathrm{i} \epsilon_{j}\right)}{q_{j-1}(v)} \frac{q_{j}\left(v+\mathrm{i} \epsilon_{j}\right)}{q_{j}(v)} \mathrm{e}^{\beta \mu_{j}} .
$$

For convenience, we have defined the functions $\phi_{ \pm}(v)=(v \pm i u)^{N / 2}$ and

$$
q_{j}(v)= \begin{cases}\phi_{-}(v) & \text { for } j=0 \\ \prod_{k_{j}=1}^{M_{j}}\left(v-v_{k_{j}}^{j}\right) & \text { for } j=1, \ldots, q-1 \\ \phi_{+}(v) & \text { for } j=q\end{cases}
$$

where the complex parameters $v_{k_{j}}^{j}$ are the so-called BA roots and $M_{j}$ is the total number of $\mathrm{BA}$ roots in set $j$. The $\mathrm{BA}$ roots have to fulfil the BA equations

$$
\frac{\lambda_{j}\left(v_{k_{j}}^{j}\right)}{\lambda_{j+1}\left(v_{k_{j}}^{j}\right)}=-1
$$

to ensure that all potential poles in the expression (15) for $\Lambda(v)$, which has to be a polynomial of degree $N$, cancel. The BA equations form a system of coupled nonlinear equations for the unknown BA roots. 


\section{Nonlinear integral equations for the $\operatorname{sl}(4)$ case}

Let us now turn to the special case of the $s l(4)$-symmetric Uimin-Sutherland model $\left(q=4, \epsilon_{j}=+1\right.$ for all $\left.j\right)$. The largest eigenvalue of the QTM lies in the sector $M_{j}=N / 2$ for all $j$. Of course, it is in principle possible to solve the BA equations (18) for some fixed Trotter number $N$. But this approach is possible only for finite $N$ and is also quite cumbersome to do numerically. As we are interested in the limit $N \rightarrow \infty$ for deriving the free energy of the model, we have to encode the BA equations into a form for which this limit can be taken analytically.

We start by defining some suitable auxiliary functions, which will in the end turn out to fulfil certain nonlinear integral equations. For convenience, we will use an abbreviated notation utilising the Yangian analogue of Young tableaux [20, 51, 52, 53]. Instead of the function $\lambda_{j}(v)$, we will write a box filled with the letter $j$,

$$
j=\lambda_{j}(v) .
$$

This corresponds to a Young tableau belonging to a vector of the first, four-dimensional fundamental representation of $s l(4)$. We also define a Young tableau belonging to the second, six-dimensional representation,

$$
\begin{array}{l|}
\hline j \\
\hline k
\end{array}=\lambda_{j}(v-\mathrm{i} / 2) \lambda_{k}(v+\mathrm{i} / 2),
$$

and for the conjugate four-dimensional representation we have

$$
\begin{array}{|c|}
\hline j \\
\hline k \\
\hline l \\
\hline
\end{array}=\lambda_{j}(v-\mathrm{i}) \lambda_{k}(v) \lambda_{l}(v+\mathrm{i}) .
$$

From fusion hierarchy [22, 25] one knows that the eigenvalues of the QTMs belonging to the three fundamental representations can be written as

$$
\begin{aligned}
& \Lambda^{(1)}(x)=1+2+3+\left.4\right|_{v=x},
\end{aligned}
$$

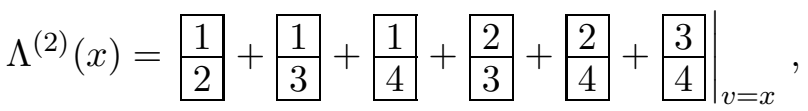

$$
\begin{aligned}
& \Lambda^{(3)}(x)=\begin{array}{|l|}
\hline 1 \\
\hline 2 \\
\hline 3 \\
\hline
\end{array}+\begin{array}{|l|}
\hline 1 \\
\hline 2 \\
\hline 4 \\
\hline
\end{array}++\begin{array}{|l|}
\hline 1 \\
\hline 3 \\
\hline 4 \\
\hline
\end{array}\left|\begin{array}{|l|}
\hline 2 \\
\hline 3 \\
\hline 4
\end{array}\right|_{v=x},
\end{aligned}
$$

where the superscripts denote the representations. In all three cases the BA equations (18) ensure that the eigenvalues are free of poles. We note that (22a) is equivalent to (15). We define the following four auxiliary functions for the first fundamental representation:

$$
b_{1}^{(1)}(x)=\left.\frac{1}{2+3+4}\right|_{v=x+\mathrm{i} / 2},
$$




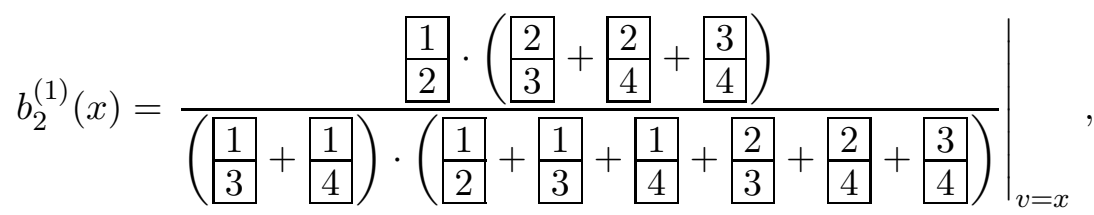

$$
\begin{aligned}
& b_{3}^{(1)}(x)=\left.\frac{\frac{1}{3} \cdot \frac{3}{4}}{\frac{1}{4} \cdot\left(\frac{1}{3}+\frac{1}{4}+\frac{2}{3}+\frac{2}{4}+\frac{3}{4}\right)}\right|_{v=x}, \\
& b_{4}^{(1)}(x)=\left.\frac{4}{1+2+3}\right|_{v=x-\mathrm{i} / 2} .
\end{aligned}
$$

We have six auxiliary functions for the second fundamental representation:

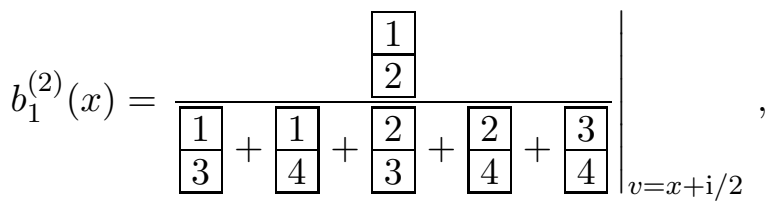

$$
\begin{aligned}
& b_{2}^{(2)}(x)=\left.\frac{1 \frac{1}{3} \cdot \frac{3}{4}}{\left(\frac{1}{4}+\frac{2}{4}+\frac{3}{4}\right) \cdot\left(\frac{2}{3}+\frac{2}{4}+\frac{3}{4}\right)}\right|_{v=x+\mathrm{i} / 2}, \\
& b_{3}^{(2)}(x)=\left.\frac{4 \cdot 4}{(2+3) \cdot(1+2+2+3+4)}\right|_{v=x},
\end{aligned}
$$

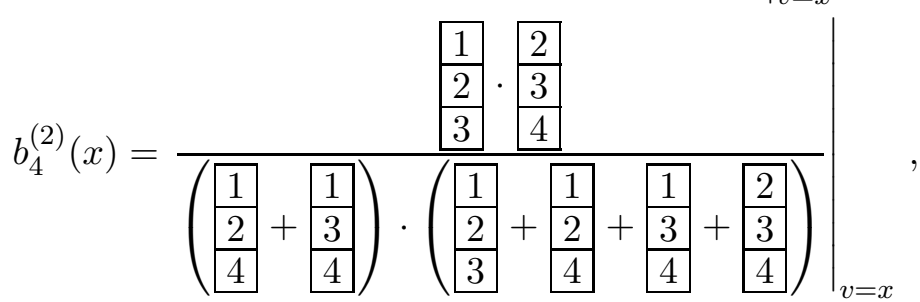

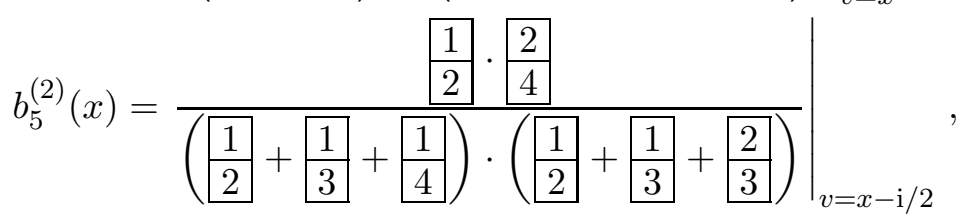

$$
\begin{aligned}
& b_{6}^{(2)}(x)=\left.\frac{\frac{3}{4}}{\frac{1}{\frac{1}{2}}+\frac{1}{3}+\frac{1}{4}+\frac{2}{3}+\frac{2}{4}}\right|_{v=x-\mathrm{i} / 2} .
\end{aligned}
$$


And finally, the four auxiliary functions for the third fundamental representation are:

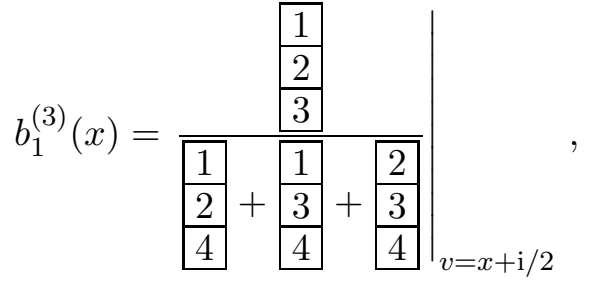

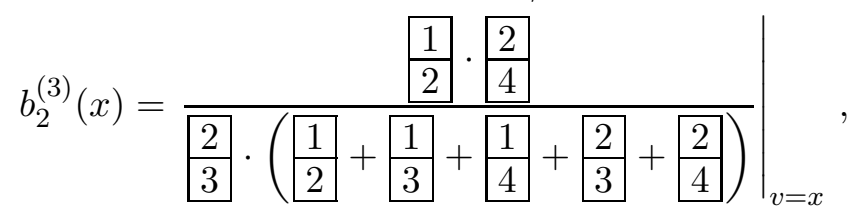

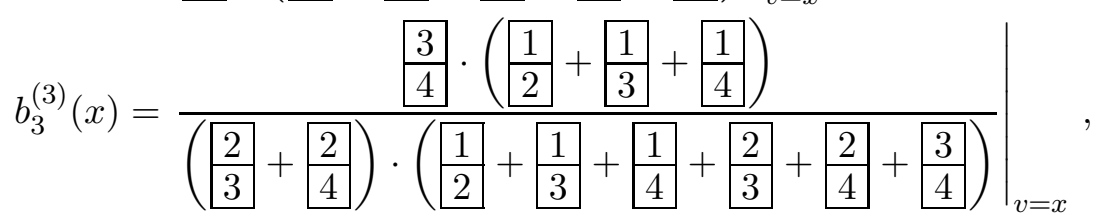

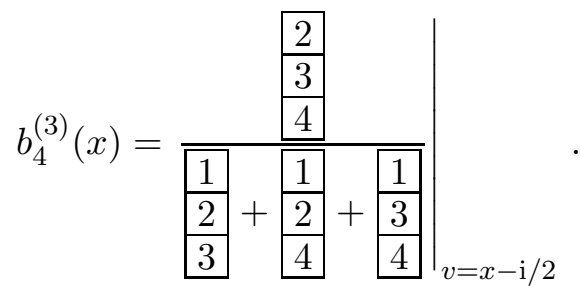

In addition to the auxiliary functions above, we define a second set of functions, namely $B_{j}^{(n)}(x)=b_{j}^{(n)}(x)+1$. These can also be written in a form, where only simple sums of Young tableaux appear as factors in the numerators and denominators, and all factors appearing in the set of functions $b_{j}^{(n)}(x)$ also appear in the set of functions $B_{j}^{(n)}(x)$. We note that all these factors are partial sums of the Young tableaux appearing in the eigenvalues (22). The set of functions (23) is related to (25) by a conjugation transformation. The set (24) is self-conjugate in this sense. Furthermore, all auxiliary functions are rational functions in terms of the spectral parameter $x$ and are analytic, non-zero and have constant asymptotics (ANZC) in a strip $-1 / 2 \lesssim \Im(x) \lesssim 1 / 2$.

Now, the actual calculation is rather straightforward but lengthy. Therefore, details are deferred to Appendix $\mathrm{A}$. The key idea is to apply a Fourier transform to the logarithmic derivative of all auxiliary functions and to exploit their analyticity properties in Fourier space. We like to stress that, although we are working with arbitrary finite $N$ throughout the derivation, in the end the limit $N \rightarrow \infty$ can be taken analytically.

We finally arrive at a system of coupled nonlinear integral equations (NLIE) of the form

$$
\mathbf{b}(x)=-\beta \boldsymbol{\epsilon}(x)-[\underline{\mathbf{K}} * \mathbf{B}](x),
$$

where we have defined

$$
\mathbf{b}=\left(\ln b_{1}^{(1)}, \ldots, \ln b_{4}^{(1)}, \ln b_{1}^{(2)}, \ldots, \ln b_{6}^{(2)}, \ln b_{1}^{(3)}, \ldots, \ln b_{4}^{(3)}\right)^{\mathrm{T}},
$$




$$
\begin{aligned}
\mathbf{B} & =\left(\ln B_{1}^{(1)}, \ldots, \ln B_{4}^{(1)}, \ln B_{1}^{(2)}, \ldots, \ln B_{6}^{(2)}, \ln B_{1}^{(3)}, \ldots, \ln B_{4}^{(3)}\right)^{\mathrm{T}} \\
\boldsymbol{\epsilon} & =\left(\epsilon_{1}^{(1)}, \ldots, \epsilon_{4}^{(1)}, \epsilon_{1}^{(2)}, \ldots, \epsilon_{6}^{(2)}, \epsilon_{1}^{(3)}, \ldots, \epsilon_{4}^{(3)}\right)^{\mathrm{T}} .
\end{aligned}
$$

Convolutions are denoted by

$$
[f * g](x)=\int_{-\infty}^{\infty} f(x-y) g(y) \frac{\mathrm{d} y}{2 \pi} .
$$

The kernel matrix $\underline{\mathbf{K}}(x)$ is a 14 by 14 matrix. As this is too large to be displayed as a whole, we divide the matrix into blocks connecting the auxiliary functions from different representations,

$$
\underline{\mathbf{K}}(x)=\left(\begin{array}{lll}
\underline{\mathbf{K}}^{(1,1)}(x) & \underline{\mathbf{K}}^{(1,2)}(x) & \underline{\mathbf{K}}^{(1,3)}(x) \\
\underline{\mathbf{K}}^{(2,1)}(x) & \underline{\mathbf{K}}^{(2,2)}(x) & \underline{\mathbf{K}}^{(2,3)}(x) \\
\underline{\mathbf{K}}^{(3,2)}(x) & \underline{\mathbf{K}}^{(3,3)}(x)
\end{array}\right) .
$$

Obviously, $\underline{\mathbf{K}}^{(1,1)}(x)$ is a 4 by 4 matrix, while $\underline{\mathbf{K}}^{(1,2)}(x)$ is a 4 by 6 matrix, etc. $\underline{\mathbf{K}}(x)$ is Hermitian and invariant under reflection along the anti-diagonal,

$$
\underline{\mathbf{K}}(x)=[\underline{\mathbf{K}}(x)]^{\dagger}, \quad[\underline{\mathbf{K}}(x)]_{j, k}=[\underline{\mathbf{K}}(x)]_{15-k, 15-j} .
$$

Therefore we only need to consider

$$
\begin{aligned}
& \underline{\mathbf{K}}^{(1,1)}(x)=\left(\begin{array}{cccc}
K_{0}(x) & K_{1}(x) & K_{1}(x) & K_{1}(x) \\
K_{2}(x) & K_{0}(x) & K_{1}(x) & K_{1}(x) \\
K_{2}(x) & K_{2}(x) & K_{0}(x) & K_{1}(x) \\
K_{2}(x) & K_{2}(x) & K_{2}(x) & K_{0}(x)
\end{array}\right) \\
& \underline{\mathbf{K}}^{(1,2)}(x)=\left(\begin{array}{llllll}
K_{11}(x) & K_{11}(x) & K_{11}(x) & K_{12}(x) & K_{12}(x) & K_{12}(x) \\
K_{11}(x) & K_{14}(x) & K_{14}(x) & K_{11}(x) & K_{11}(x) & K_{12}(x) \\
K_{13}(x) & K_{11}(x) & K_{14}(x) & K_{11}(x) & K_{14}(x) & K_{11}(x) \\
K_{13}(x) & K_{13}(x) & K_{11}(x) & K_{13}(x) & K_{11}(x) & K_{11}(x)
\end{array}\right) \text {, } \\
& \underline{\mathbf{K}}^{(1,3)}(x)=\left(\begin{array}{llll}
K_{15}(x) & K_{15}(x) & K_{15}(x) & K_{16}(x) \\
K_{15}(x) & K_{15}(x) & K_{18}(x) & K_{15}(x) \\
K_{15}(x) & K_{19}(x) & K_{15}(x) & K_{15}(x) \\
K_{17}(x) & K_{15}(x) & K_{15}(x) & K_{15}(x)
\end{array}\right) \\
& \underline{\mathbf{K}}^{(2,2)}(x)=\left(\begin{array}{cccccc}
K_{3}(x) & K_{4}(x) & K_{4}(x) & K_{4}(x) & K_{4}(x) & K_{6}(x) \\
K_{5}(x) & K_{3}(x) & K_{4}(x) & K_{4}(x) & K_{8}(x) & K_{4}(x) \\
K_{5}(x) & K_{5}(x) & K_{3}(x) & K_{10}(x) & K_{4}(x) & K_{4}(x) \\
K_{5}(x) & K_{5}(x) & K_{10}(x) & K_{3}(x) & K_{4}(x) & K_{4}(x) \\
K_{5}(x) & K_{9}(x) & K_{5}(x) & K_{5}(x) & K_{3}(x) & K_{4}(x) \\
K_{7}(x) & K_{5}(x) & K_{5}(x) & K_{5}(x) & K_{5}(x) & K_{3}(x)
\end{array}\right) .
\end{aligned}
$$

The remaining matrices easily follow from the relations

$$
\underline{\mathbf{K}}^{(3,3)}(x)=\underline{\mathbf{K}}^{(1,1)}(x), \quad \underline{\mathbf{K}}^{(2,1)}(x)=\left[\underline{\mathbf{K}}^{(1,2)}(x)\right]^{\dagger},
$$




$$
\begin{aligned}
\underline{\mathbf{K}}^{(3,1)}(x) & =\left[\underline{\mathbf{K}}^{(1,3)}(x)\right]^{\dagger}, & \underline{\mathbf{K}}^{(3,2)}(x)=\left[\underline{\mathbf{K}}^{(2,3)}(x)\right]^{\dagger}, \\
{\left[\underline{\mathbf{K}}^{(2,3)}(x)\right]_{j, k} } & =\left[\underline{\mathbf{K}}^{(1,2)}(x)\right]_{7-k, 5-j} . &
\end{aligned}
$$

The kernels are defined as $K_{j}(x)=\int_{-\infty}^{\infty} \widehat{K}_{j}(k) \mathrm{e}^{\mathrm{i} k x} \mathrm{~d} k$, where

$$
\begin{aligned}
\widehat{K}_{0}(k) & =\widehat{K}_{[4]}^{(1,1)}(k), & \widehat{K}_{1}(k) & =\widehat{K}_{[4]}^{(1,1)}(k)+\mathrm{e}^{-k / 2-|k| / 2}, \\
\widehat{K}_{2}(k) & =\widehat{K}_{[4]}^{(1,1)}(k)+\mathrm{e}^{k / 2-|k| / 2}, & \widehat{K}_{3}(k) & =\widehat{K}_{[4]}^{(2,2)}(k), \\
\widehat{K}_{4}(k) & =\widehat{K}_{[4]}^{(2,2)}(k)+\mathrm{e}^{-k / 2-|k| / 2}, & \widehat{K}_{5}(k) & =\widehat{K}_{[4]}^{(2,2)}(k)+\mathrm{e}^{k / 2-|k| / 2}, \\
\widehat{K}_{6}(k) & =\widehat{K}_{[4]}^{(2,2)}(k)+\mathrm{e}^{-k-|k|}, & \widehat{K}_{7}(k) & =\widehat{K}_{[4]}^{(2,2)}(k)+\mathrm{e}^{k-|k|}, \\
\widehat{K}_{8}(k) & =\widehat{K}_{[4]}^{(2,2)}(k)+2 \mathrm{e}^{-k / 2-|k| / 2}, & \widehat{K}_{9}(k) & =\widehat{K}_{[4]}^{(2,2)}(k)+2 \mathrm{e}^{k / 2-|k| / 2}, \\
\widehat{K}_{10}(k) & =\widehat{K}_{[4]}^{(2,2)}(k)+\mathrm{e}^{-|k|}, & \widehat{K}_{11}(k) & =\widehat{K}_{[4]}^{(1,2)}(k), \\
\widehat{K}_{12}(k) & =\widehat{K}_{[4]}^{(1,2)}(k)+\mathrm{e}^{-k-|k| / 2}-\mathrm{e}^{-k / 2}, & \widehat{K}_{13}(k) & =\widehat{K}_{[4]}^{(1,2)}(k)+\mathrm{e}^{k-|k| / 2}-\mathrm{e}^{k / 2}, \\
\widehat{K}_{14}(k) & =\widehat{K}_{[4]}^{(1,2)}(k)+\mathrm{e}^{-|k| / 2}, & \widehat{K}_{15}(k) & =\widehat{K}_{[4]}^{(1,3)}(k), \\
\widehat{K}_{16}(k) & =\widehat{K}_{[4]}^{(1,3)}(k)+\mathrm{e}^{-3 k / 2-|k| / 2}-\mathrm{e}^{-k}, & \widehat{K}_{17}(k) & =\widehat{K}_{[4]}^{(1,3)}(k)+\mathrm{e}^{3 k / 2-|k| / 2}-\mathrm{e}^{k}, \\
\widehat{K}_{18}(k) & =\widehat{K}_{[4]}^{(1,3)}(k)+\mathrm{e}^{-k / 2-|k| / 2}-1, & \widehat{K}_{19}(k) & =\widehat{K}_{[4]}^{(1,3)}(k)+\mathrm{e}^{k / 2-|k| / 2},
\end{aligned}
$$

with the function

$$
\widehat{K}_{[q]}^{(n, m)}(k)=\mathrm{e}^{|k| / 2} \frac{\sinh (\min (n, m) k / 2) \sinh ([q-\max (n, m)] k / 2)}{\sinh (k / 2) \sinh (q k / 2)}-\delta_{n m} .
$$

We note that in spectral parameter space all kernels can be written in terms of digamma and simple rational functions. Nevertheless our notation is more useful here as the numerical treatment of the NLIE can be conveniently done in Fourier space. The functions $K_{[q]}^{(n, m)}(x)=\int_{-\infty}^{\infty} \widehat{K}_{[q]}^{(n, m)}(k) \mathrm{e}^{\mathrm{i} k x} \mathrm{~d} k$ are related to the $S$-matrix of elementary excitations [54] via

$$
K_{[q]}^{(n, m)}(x)=\frac{\mathrm{d}}{\mathrm{d} x}\left[\mathrm{i} \ln S_{[q]}^{(n, m)}(x)\right] .
$$

The bare energies in (29) are $\epsilon_{j}^{(n)}(x)=V_{[4]}^{(n)}(x)+c_{j}^{(n)}$, where

$$
V_{[q]}^{(n)}(x)=\frac{2 \pi}{q} \frac{\sin (\pi n / q)}{\cosh (2 \pi x / q)-\cos (\pi n / q)},
$$

and the constants are given by

$$
\begin{array}{ll}
c_{1}^{(1)}=\left(-3 \mu_{1}+\mu_{2}+\mu_{3}+\mu_{4}\right) / 4, & c_{2}^{(1)}=\left(\mu_{1}-3 \mu_{2}+\mu_{3}+\mu_{4}\right) / 4, \\
c_{3}^{(1)}=\left(\mu_{1}+\mu_{2}-3 \mu_{3}+\mu_{4}\right) / 4, & c_{4}^{(1)}=\left(\mu_{1}+\mu_{2}+\mu_{3}-3 \mu_{4}\right) / 4, \\
c_{1}^{(2)}=\left(-\mu_{1}-\mu_{2}+\mu_{3}+\mu_{4}\right) / 2, & c_{2}^{(2)}=\left(-\mu_{1}+\mu_{2}-\mu_{3}+\mu_{4}\right) / 2,
\end{array}
$$




$$
\begin{array}{ll}
c_{3}^{(2)}=\left(-\mu_{1}+\mu_{2}+\mu_{3}-\mu_{4}\right) / 2, & c_{4}^{(2)}=\left(\mu_{1}-\mu_{2}-\mu_{3}+\mu_{4}\right) / 2, \\
c_{5}^{(2)}=\left(\mu_{1}-\mu_{2}+\mu_{3}-\mu_{4}\right) / 2, & c_{6}^{(2)}=\left(\mu_{1}+\mu_{2}-\mu_{3}-\mu_{4}\right) / 2, \\
c_{1}^{(3)}=\left(-\mu_{1}-\mu_{2}-\mu_{3}+3 \mu_{4}\right) / 4, & c_{2}^{(3)}=\left(-\mu_{1}-\mu_{2}+3 \mu_{3}-\mu_{4}\right) / 4, \\
c_{3}^{(3)}=\left(-\mu_{1}+3 \mu_{2}-\mu_{3}-\mu_{4}\right) / 4, & c_{4}^{(3)}=\left(3 \mu_{1}-\mu_{2}-\mu_{3}-\mu_{4}\right) / 4 .
\end{array}
$$

Finally, the largest eigenvalue of the QTM can be written in terms of the auxiliary functions,

$$
\ln \Lambda_{\max }(0)=-\beta\left(1-\frac{\pi}{4}-\frac{3}{2} \ln 2-\frac{1}{4} \sum_{j=1}^{4} \mu_{j}\right)+\sum_{n=1}^{3} \sum_{j=1}^{d_{n}}\left[V_{[4]}^{(n)} * \ln B_{j}^{(n)}\right](0),
$$

where $d_{n}=\left(\begin{array}{l}4 \\ n\end{array}\right)$ is the dimension of the $n$th fundamental representation. Therefore, the problem of solving the infinitely many BA equations (18) in the limit $N \rightarrow \infty$ has been reduced to finding a finite set of functions satisfying the NLIE (26)-(39). The NLIE is valid for arbitrary finite temperature and chemical potentials.

\section{Analytical investigation}

\subsection{Investigation of the $\operatorname{sl}(3)$ limit}

First we want to show how our formulation (26) -(40) reduces to the known NLIE for the $s l(3)$-symmetric case [33] by freezing out one of the states. We choose the state $\alpha=4$ and accordingly treat the limit $\mu_{4} \rightarrow-\infty$. We observe that

$$
\begin{array}{llll}
b_{1}^{(1)}(x) & =\mathcal{O}(1), & b_{2}^{(1)}(x)=\mathcal{O}(1), & b_{3}^{(1)}(x)=\mathcal{O}(1), \\
b_{4}^{(1)}(x)=\mathcal{O}\left(\mathrm{e}^{\beta \mu_{4}}\right), & b_{1}^{(2)}(x)=\mathcal{O}(1), & b_{2}^{(2)}(x)=\mathcal{O}(1), \\
b_{3}^{(2)}(x)=\mathcal{O}\left(\mathrm{e}^{\beta \mu_{4}}\right), & b_{4}^{(2)}(x)=\mathcal{O}(1), & b_{5}^{(2)}(x)=\mathcal{O}\left(\mathrm{e}^{\beta \mu_{4}}\right), \\
b_{6}^{(2)}(x)=\mathcal{O}\left(\mathrm{e}^{\beta \mu_{4}}\right), & b_{1}^{(3)}(x)=\mathcal{O}\left(\mathrm{e}^{-\beta \mu_{4}}\right), & b_{2}^{(3)}(x)=\mathcal{O}\left(\mathrm{e}^{\beta \mu_{4}}\right), \\
b_{3}^{(3)}(x)=\mathcal{O}\left(\mathrm{e}^{\beta \mu_{4}}\right), & b_{4}^{(3)}(x)=\mathcal{O}\left(\mathrm{e}^{\beta \mu_{4}}\right) . &
\end{array}
$$

Therefore only seven of the auxiliary functions survive. We can regard

$$
b_{4}^{(1)}(x) \equiv b_{3}^{(2)}(x) \equiv b_{5}^{(2)}(x) \equiv b_{6}^{(2)}(x) \equiv b_{2}^{(3)}(x) \equiv b_{3}^{(3)}(x) \equiv b_{4}^{(3)}(x) \equiv 0 .
$$

We also conclude that $b_{1}^{(3)}(x) / B_{1}^{(3)}(x) \rightarrow 1$. Using this information, the equation for $\ln b_{1}^{(3)}(x)$ linearises and can be solved analytically. We get

$$
\begin{aligned}
\ln B_{1}^{(3)}(x)=-\beta & \left(W(x)-\frac{\mu_{1}+\mu_{2}+\mu_{3}}{3}+\mu_{4}\right) \\
& -\left[V_{[3]}^{(2)} * \ln \left(B_{1}^{(1)} B_{2}^{(1)} B_{3}^{(1)}\right)\right](x)-\left[V_{[3]}^{(1)} * \ln \left(B_{1}^{(2)} B_{2}^{(2)} B_{4}^{(2)}\right)\right](x),
\end{aligned}
$$


where

$$
W(x)=\int_{-\infty}^{\infty} \frac{\mathrm{e}^{-|k| / 2}}{\mathrm{e}^{-k}+1+\mathrm{e}^{k}} \mathrm{e}^{\mathrm{i} k x} \mathrm{~d} k
$$

Substituting this into our NLIE and relabelling $b_{4}^{(2)}(x)$ to $b_{3}^{(2)}(x)$, we are again left with a NLIE of type (26), but with only six remaining auxiliary functions belonging to the two fundamental representations of $s l(3)$. Here we get the kernel matrix

$$
\underline{\mathbf{K}}(x)=\left(\begin{array}{ll}
\underline{\mathbf{K}}^{(1,1)}(x) & \underline{\mathbf{K}}^{(1,2)}(x) \\
\underline{\mathbf{K}}^{(1,2)}(x) & \underline{\mathbf{K}}^{(1,1)}(x)
\end{array}\right),
$$

where the submatrices are given by

$$
\begin{aligned}
\underline{\mathbf{K}}^{(1,1)}(x) & =\left(\begin{array}{lll}
K_{0}(x) & K_{1}(x) & K_{1}(x) \\
K_{2}(x) & K_{0}(x) & K_{1}(x) \\
K_{2}(x) & K_{2}(x) & K_{0}(x)
\end{array}\right), \\
\underline{\mathbf{K}}^{(1,2)}(x) & =\left(\begin{array}{lll}
K_{3}(x) & K_{3}(x) & K_{4}(x) \\
K_{3}(x) & K_{6}(x) & K_{3}(x) \\
K_{5}(x) & K_{3}(x) & K_{3}(x)
\end{array}\right) .
\end{aligned}
$$

The Fourier transformed kernels are found to be

$$
\begin{aligned}
\widehat{K}_{0}(k) & =\widehat{K}_{[3]}^{(1,1)}(k), & \widehat{K}_{1}(k) & =\widehat{K}_{[3]}^{(1,1)}(k)+\mathrm{e}^{-k / 2-|k| / 2}, \\
\widehat{K}_{2}(k) & =\widehat{K}_{[3]}^{(1,1)}(k)+\mathrm{e}^{k / 2-|k| / 2}, & \widehat{K}_{3}(k) & =\widehat{K}_{[3]}^{(1,2)}(k), \\
\widehat{K}_{4}(k) & =\widehat{K}_{[3]}^{(1,2)}(k)+\mathrm{e}^{-k-|k| / 2}-\mathrm{e}^{-k / 2}, & \widehat{K}_{5}(k) & =\widehat{K}_{[3]}^{(1,2)}(k)+\mathrm{e}^{k-|k| / 2}-\mathrm{e}^{k / 2}, \\
\widehat{K}_{6}(k) & =\widehat{K}_{[3]}^{(1,2)}(k)+\mathrm{e}^{-|k| / 2} . & &
\end{aligned}
$$

For the bare energies, we get $\epsilon_{j}^{(n)}(x)=V_{[3]}^{(n)}(x)+c_{j}^{(n)}$ with the constants

$$
\begin{array}{ll}
c_{1}^{(1)}=\left(-2 \mu_{1}+\mu_{2}+\mu_{3}\right) / 3, & c_{2}^{(1)}=\left(\mu_{1}-2 \mu_{2}+\mu_{3}\right) / 3, \\
c_{3}^{(1)}=\left(\mu_{1}+\mu_{2}-2 \mu_{3}\right) / 3, & c_{1}^{(2)}=\left(-\mu_{1}-\mu_{2}+2 \mu_{3}\right) / 3, \\
c_{2}^{(2)}=\left(-\mu_{1}+2 \mu_{2}-\mu_{3}\right) / 3, & c_{3}^{(2)}=\left(2 \mu_{1}-\mu_{2}-\mu_{3}\right) / 3 .
\end{array}
$$

The largest eigenvalue is finally given by

$$
\ln \Lambda_{\max }(0)=-\beta\left(1-\frac{\pi}{3 \sqrt{3}}-\ln 3-\frac{1}{3} \sum_{j=1}^{3} \mu_{j}\right)+\sum_{n=1}^{2} \sum_{j=1}^{3}\left[V_{[3]}^{(n)} * \ln B_{j}^{(n)}\right](0) .
$$

As expected, this is exactly the known NLIE for the $\operatorname{sl}(3)$-symmetric case [33].

We can also recover the explicit form of all auxiliary functions for the $s l(3)$ case. We drop all Young tableaux that contain 4 in the auxiliary functions (23)-(25) as $\lambda_{4}(v) \rightarrow 0$ in the limit $\mu_{4} \rightarrow-\infty$. We have already seen that seven of the functions 
become zero, while one function diverges. After relabelling $b_{4}^{(2)}(x)$ to $b_{3}^{(2)}(x)$ again, the remaining six functions take the form

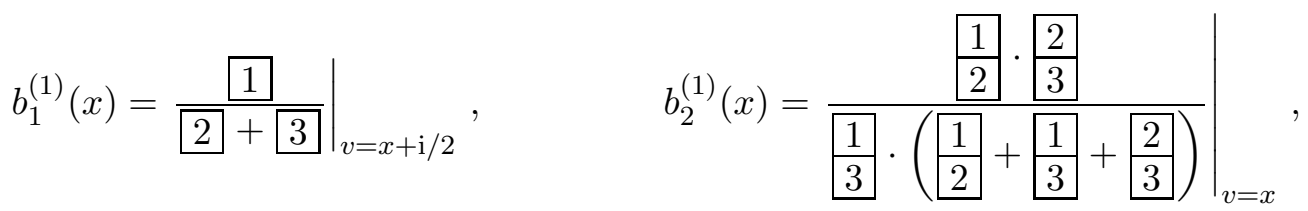

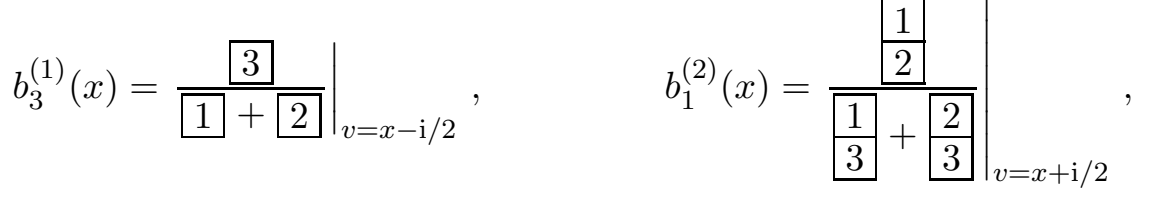

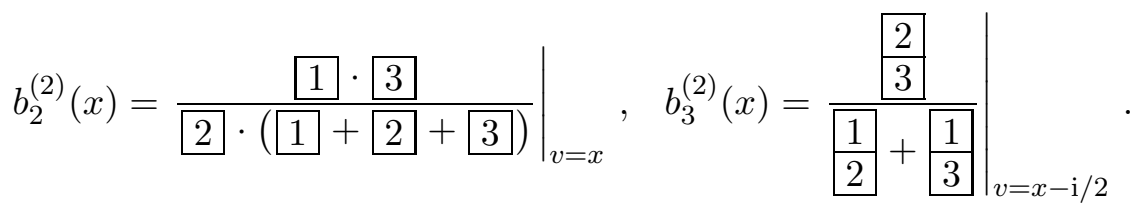

Although only two sets of BA roots are present in the $s l(3)$ case, these auxiliary functions still contain the function $q_{3}(v)$, which enters through $\lambda_{3}(v)$. But, as already indicated by the definition of $q_{3}(v)$ for the case $q=3$ in (17), we have to demand $q_{3}(v)=\phi_{+}(v)$ in the limit $\mu_{4} \rightarrow-\infty$ in order that the previously derived NLIE can also be derived directly from the auxiliary functions (50). The auxiliary functions are equal to those presented in [33].

We finally note that our choice of freezing out the state $\alpha=4$ is completely arbitrary. Choosing one of the other states yields, after relabelling some of the indices, the same NLIE and auxiliary functions. For $\mu_{1} \rightarrow-\infty$, we find that $q_{1}(v)=\phi_{-}(v)$, while $q_{2}(v)$ and $q_{3}(v)$ contain the remaining BA roots. For $\mu_{\alpha} \rightarrow-\infty$ with $\alpha=2$ or 3 , we find that $q_{\alpha-1}(v)=q_{\alpha}(v)$.

\subsection{Limit $T \rightarrow 0$ and critical fields}

We divide the NLIE (26) by $\beta$ and define rescaled auxiliary functions by

$$
e_{j}^{(n)}(x)=\frac{1}{\beta} \ln b_{j}^{(n)}(x), \quad E_{j}^{(n)}(x)=\frac{1}{\beta} \ln B_{j}^{(n)}(x) .
$$

In the limit $T \rightarrow 0(\beta \rightarrow \infty)$ we get

$$
E_{j}^{(n)}(x) \rightarrow e_{j}^{+(n)}(x)=\left\{\begin{array}{ll}
e_{j}^{(n)}(x) & \text { if } \Re\left(e_{j}^{(n)}(x)\right)>0 \\
0 & \text { if } \Re\left(e_{j}^{(n)}(x)\right) \leq 0
\end{array} .\right.
$$

Obviously, auxiliary functions with negative real parts for all $x \in \mathbb{R}$ do no longer contribute to the ground-state energy, because $e_{j}^{+(n)}(x) \equiv 0$ for these functions.

Without loss of generality, we choose the chemical potentials to be ordered, $\mu_{1} \geq$ $\mu_{2} \geq \mu_{3} \geq \mu_{4}$. Changing the order just amounts to some permutation of indices in the following calculations. In our case, we observe that the only remaining auxiliary functions 
are $e_{1}^{(n)}(x)$ for $n=1,2,3$, i.e. one from every representation. The NLIE linearise and take the form

$$
e_{1}^{(n)}(x)=-V_{[4]}^{(n)}(x)-c_{1}^{(n)}-\sum_{m=1}^{3}\left[K_{[4]}^{(n, m)} * e_{1}^{+(m)}\right](x) \quad(n=1,2,3) .
$$

It follows that the remaining auxiliary functions are real and symmetric with respect to the spectral parameter. The ground-state energy is given by

$$
f_{0}=1-\frac{\pi}{4}-\frac{3}{2} \ln 2-\frac{1}{4} \sum_{j=1}^{4} \mu_{j}-\sum_{n=1}^{3}\left[V_{[4]}^{(n)} * e_{1}^{+(n)}\right](0) .
$$

These equations have a particularly simple solution if all chemical potentials are equal, $\mu_{1}=\mu_{2}=\mu_{3}=\mu_{4}$. In this case, we get $e_{1}^{(n)}(x)=-V_{[4]}^{(n)}(x)$ and $e_{1}^{+(n)}(x) \equiv 0$ for all $n$. Therefore, the ground-state energy is just $f_{0}=1-\pi / 4-3 \ln (2) / 2$.

In general, depending on certain differences of the chemical potentials, the ground state can be in one of four possible phases. We start with the phase, where all degrees of freedom are frozen out, i.e. only the state $\alpha=1$ survives. In this case, we have $e_{1}^{(n)}(x)=e_{1}^{+(n)}(x)$ for all $n$. As a consequence, (53) can be solved analytically. We find the restriction $\mu_{1}-\mu_{2} \geq 4$ and obtain

$$
e_{1}^{(1)}(x)=\mu_{1}-\mu_{2}-\frac{4}{4 x^{2}+1}, \quad e_{1}^{(2)}(x)=\mu_{2}-\mu_{3}, \quad e_{1}^{(3)}(x)=\mu_{3}-\mu_{4},
$$

while the ground-state energy turns out to be $f_{0}=1-\mu_{1}$. As expected, the ground state is fully polarised. We call the point $\mu_{1}-\mu_{2}=4$ the first critical field.

Below the first critical field, i.e. if $\mu_{1}-\mu_{2}<4$, the function $e_{1}^{(1)}(x)$ possesses two symmetrically distributed real roots, and we have $e_{1}^{(n)}(x)=e_{1}^{+(n)}(x)$ only for $n=2,3$. We can still solve (53) for the latter two functions and get

$$
\begin{aligned}
& e_{1}^{(1)}(x)=-V_{[2]}^{(1)}(x)+\frac{\mu_{1}-\mu_{2}}{2}-\left[K_{[2]}^{(1,1)} * e_{1}^{+(1)}\right](x), \\
& e_{1}^{(2)}(x)=K_{[2]}^{(1,1)}(x)+\frac{1}{2} \sum_{j=1}^{2}\left(\mu_{j}-\mu_{3}\right)-\left[V_{[2]}^{(1)} * e_{1}^{+(1)}\right](x), \\
& e_{1}^{(3)}(x)=\mu_{3}-\mu_{4} .
\end{aligned}
$$

For the ground-state energy, we arrive at

$$
f_{0}=1-2 \ln 2-\frac{\mu_{1}+\mu_{2}}{2}-\left[V_{[2]}^{(1)} * e_{1}^{+(1)}\right](x) .
$$

This is exactly the $T=0$ behaviour of the spin- $1 / 2$ Heisenberg chain. Two states, $\alpha=1$ and 2 , are present in the ground state. Note that these equations are valid only above the second critical field, i.e. as long as $e_{1}^{(2)}(x) \geq 0$ for all $x \in \mathbb{R}$. From (56b), we find the restriction

$$
\sum_{j=1}^{2}\left(\mu_{j}-\mu_{3}\right) \geq 4 \ln 2+2 \cdot\left[V_{[2]}^{(1)} * e_{1}^{+(1)}\right](0)
$$


where the positive convolution term unfortunately still depends on the function $e_{1}^{+(1)}(x)$, which is not explicitly known. The convolution term vanishes if $\mu_{1}=\mu_{2}$.

Below the second critical field, the state $\alpha=3$ also contributes to the ground state. Both $e_{1}^{(1)}(x)$ and $e_{1}^{(2)}(x)$ possess two real roots, and only $e_{1}^{(3)}(x)=e^{+(3)}(x)$ remains valid. Here, we recover the $T=0$ behaviour of the $s l(3)$-symmetric US model,

$$
\begin{aligned}
& e_{1}^{(n)}(x)=-V_{[3]}^{(n)}(x)-c_{1}^{(n)}-\sum_{m=1}^{2}\left[K_{[3]}^{(n, m)} * e_{1}^{+(m)}\right](x) \quad(n=1,2), \\
& e_{1}^{(3)}(x)=-W(x)+\frac{1}{3} \sum_{j=1}^{3}\left(\mu_{j}-\mu_{4}\right)-\sum_{n=1}^{2}\left[V_{[3]}^{(3-n)} * e_{1}^{+(n)}\right](x),
\end{aligned}
$$

where $c_{1}^{(n)}$ are the constants of the $\operatorname{sl}(3)$ case defined in (48). The ground-state energy can be calculated by use of

$$
f_{0}=1-\frac{\pi}{3 \sqrt{3}}-\ln 3-\frac{1}{3} \sum_{j=1}^{3} \mu_{j}-\sum_{n=1}^{2}\left[V_{[3]}^{(n)} * e_{1}^{+(n)}\right](0) .
$$

These equations hold as long as we are above the third and last critical field. We see from (59b) that the restriction is

$$
\sum_{j=1}^{3}\left(\mu_{j}-\mu_{4}\right) \geq \pi \sqrt{3}-3 \ln 3+3 \cdot \sum_{n=1}^{2}\left[V_{[3]}^{(3-n)} * e_{1}^{+(n)}\right](0),
$$

where we again have no explicit expression for the positive convolution terms, which vanish if $\mu_{1}=\mu_{2}=\mu_{3}$.

Finally, below the third and last critical field, all auxiliary functions possess two symmetrically distributed real roots. Therefore, equations (53) and (54) have to be used without further simplification. In this case, all four states contribute to the ground state. We note that the equations (53) and (54) are equal to those one can get either from the traditional TBA equations in the limit $T \rightarrow 0$ or directly from the BA equations for the Hamiltonian [13].

\section{Numerical investigation}

The NLIE of the $s l(4)$-symmetric Uimin-Sutherland model (26) are of a type that allows for an efficient numerical solution by iteration and use of the fast Fourier transform (FFT) to calculate the convolutions. As initial values, some discretised functions $\ln B_{j}^{(n)}(x)$ are taken. The FFT is applied and the right-hand sides of the NLIE are computed in Fourier space. After that, the functions $\ln b_{j}^{(n)}(x)$ are obtained using the inverse FFT to eventually yield a new approximation for the functions $\ln B_{j}^{(n)}(x)$. These steps have to be repeated, each time starting with the previous approximation for $\ln B_{j}^{(n)}(x)$, until the numerical error is small enough. Finally, the free energy is calculated using (14) and (40). 
From our NLIE it is also possible to directly calculate derivatives of the free energy with respect to some parameter $p$. We just have to consider the corresponding derivatives of the NLIE and of the expression for the eigenvalue. For the first derivative, we exploit the relation

$$
\frac{\partial}{\partial p} \ln B_{j}^{(n)}(x)=\frac{b_{j}^{(n)}(x)}{B_{j}^{(n)}(x)} \cdot \frac{\partial}{\partial p} \ln b_{j}^{(n)}(x)
$$

and use an additional relation for the calculation of the second derivative,

$$
\frac{\partial^{2}}{\partial p^{2}} \ln B_{j}^{(n)}(x)=\frac{b_{j}^{(n)}(x)}{B_{j}^{(n)}(x)}\left\{\frac{1}{B_{j}^{(n)}(x)}\left(\frac{\partial}{\partial p} \ln b_{j}^{(n)}(x)\right)^{2}+\frac{\partial^{2}}{\partial p^{2}} \ln b_{j}^{(n)}(x)\right\} .
$$

In this case, the numerical calculation is done step by step. After solving the unmodified NLIE to obtain $\ln B_{j}^{(n)}(x)$, its first and finally its second derivative are solved in an analogous way. In each step, results from the previous calculations are used.

In the following, we will provide some numerical results for a special application of the one-dimensional sl(4)-symmetric Uimin-Sutherland model. We consider the Hamiltonian

$$
\mathcal{H}=\sum_{j=1}^{L}\left(2 \boldsymbol{S}_{j} \boldsymbol{S}_{j+1}+1 / 2\right)\left(2 \boldsymbol{\tau}_{j} \boldsymbol{\tau}_{j+1}+1 / 2\right)-\sum_{j=1}^{L}\left(g_{S} h S_{j}^{z}+g_{\tau} h \tau_{j}^{z}\right),
$$

which describes the $S U(4)$-symmetric case of a $S U(2) \times S U(2)$ spin-orbital model at the supersymmetric point. $\boldsymbol{S}_{j}$ is a $S U(2)$ spin-1/2 operator acting on the spins and $\boldsymbol{\tau}_{j}$ is a $S U(2)$ spin-1/2 operator acting on the orbital pseudo-spin degrees of freedom. We have allowed for an external magnetic field $h$, which couples to the spins and orbital pseudospins with Landé factors $g_{S}$ and $g_{\tau}$, respectively. Clearly, the Hamiltonian is equivalent to the $s l(4)$-symmetric US Hamiltonian (6) if we use the basis

$$
|1\rangle=\left|\uparrow_{S} \uparrow_{\tau}\right\rangle, \quad|2\rangle=\left|\uparrow_{S} \downarrow_{\tau}\right\rangle, \quad|3\rangle=\left|\downarrow_{S} \uparrow_{\tau}\right\rangle, \quad|4\rangle=\left|\downarrow_{S} \downarrow_{\tau}\right\rangle
$$

and accordingly set

$$
\begin{array}{ll}
\mu_{1}=\left(g_{S}+g_{\tau}\right) h / 2, & \mu_{2}=\left(g_{S}-g_{\tau}\right) h / 2, \\
\mu_{3}=-\left(g_{S}-g_{\tau}\right) h / 2, & \mu_{4}=-\left(g_{S}+g_{\tau}\right) h / 2 .
\end{array}
$$

We are mainly interested in the entropy $S$, specific heat $C$, magnetisation $M$ and magnetic susceptibility $\chi$, which are defined by

$$
S=-\frac{\partial f}{\partial T}, \quad C=-T \frac{\partial^{2} f}{\partial T^{2}}, \quad M=-\frac{\partial f}{\partial h}, \quad \chi=-\frac{\partial^{2} f}{\partial h^{2}} .
$$

In Figure 2 on the next page and Figure 3 on page 18, results are shown for the case $g_{S}=1$ and $g_{\tau}=0$, for which the magnetic field couples only to the spins. In this case, we have $\mu_{1}=\mu_{2}=h / 2$ and $\mu_{3}=\mu_{4}=-h / 2$. Therefore we know from our analytical investigation that there is only one critical field exactly at $h_{c}=2 \ln 2 \approx 1.39$. Below 


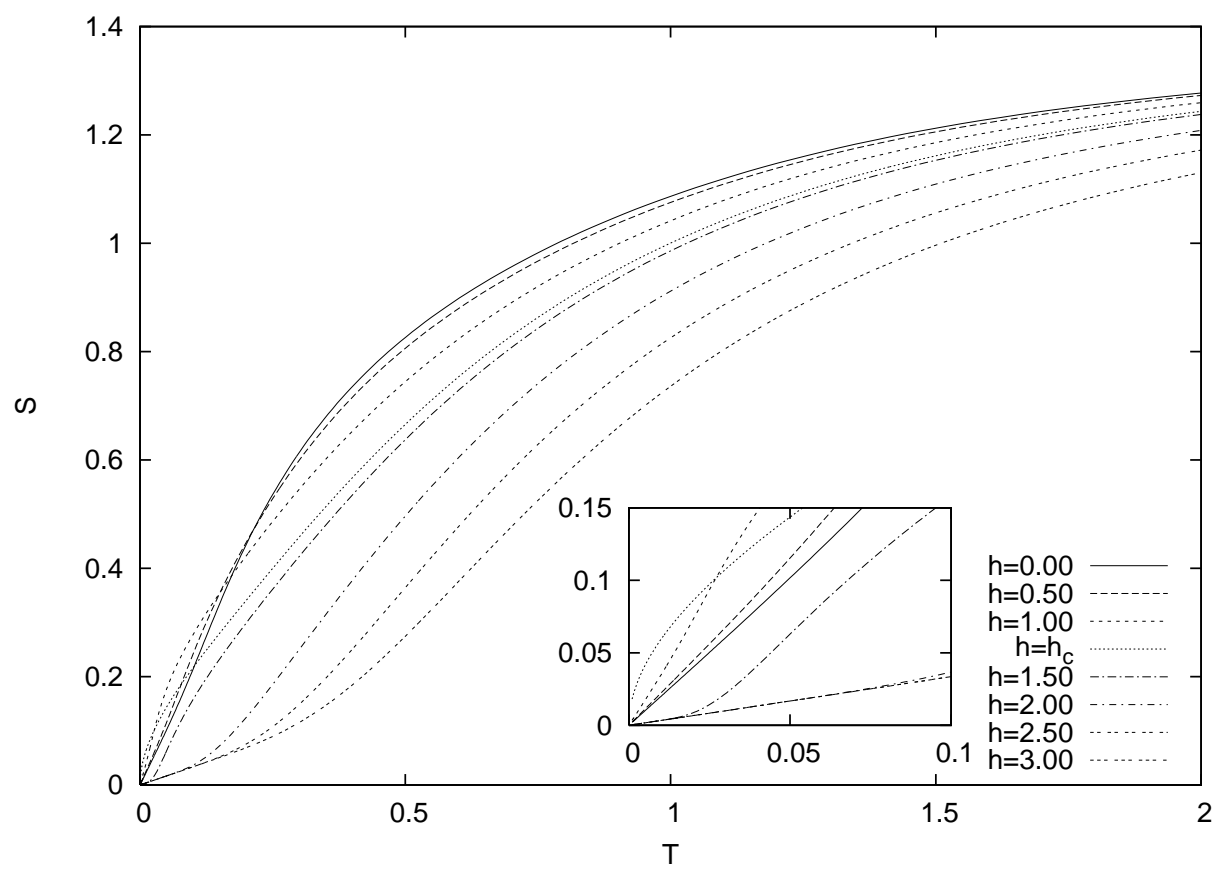

(a) Entropy vs. temperature.

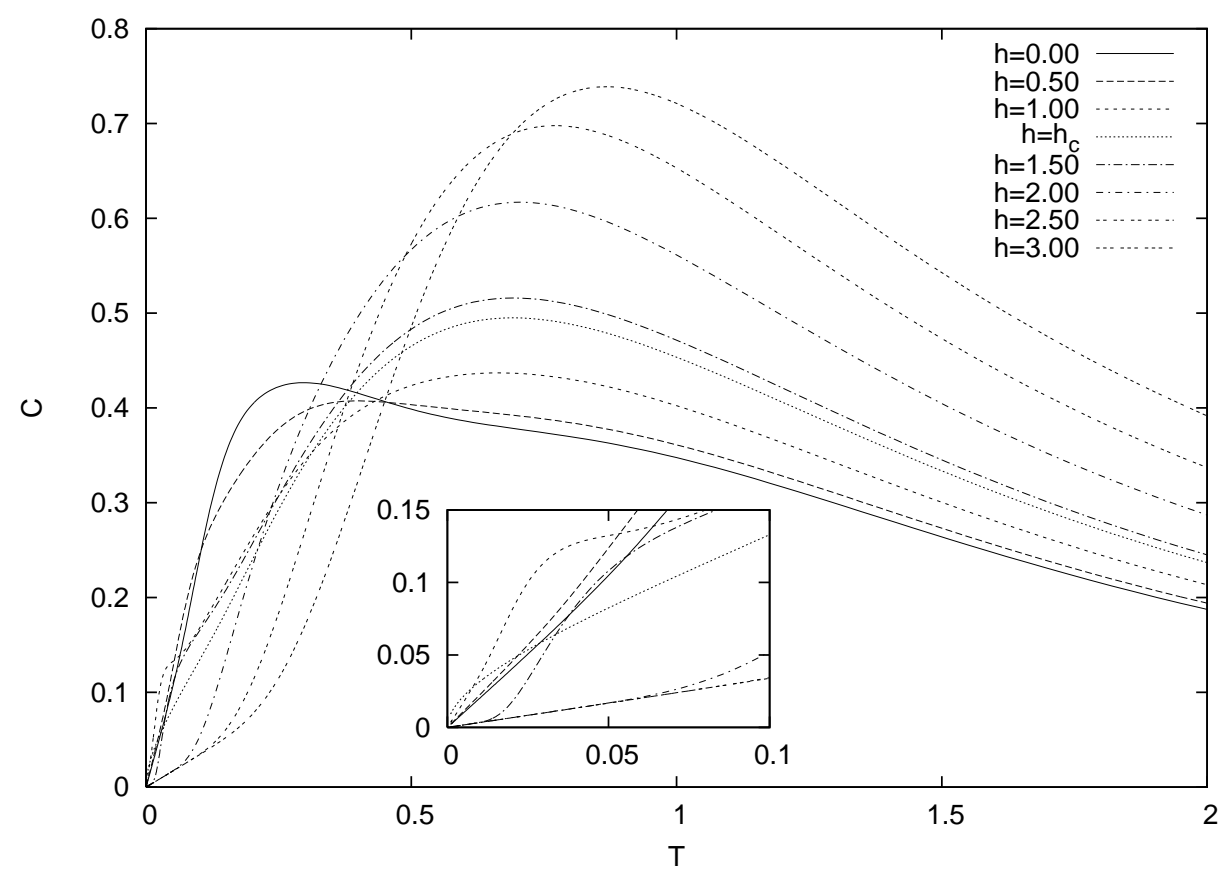

(b) Specific heat vs. temperature.

Figure 2: Entropy and specific heat of the spin-orbital model at $g_{S}=1, g_{\tau}=0$ for various magnetic fields. The insets show the low-temperature parts. The critical field is $h_{c}=2 \ln 2 \approx 1.39$. 


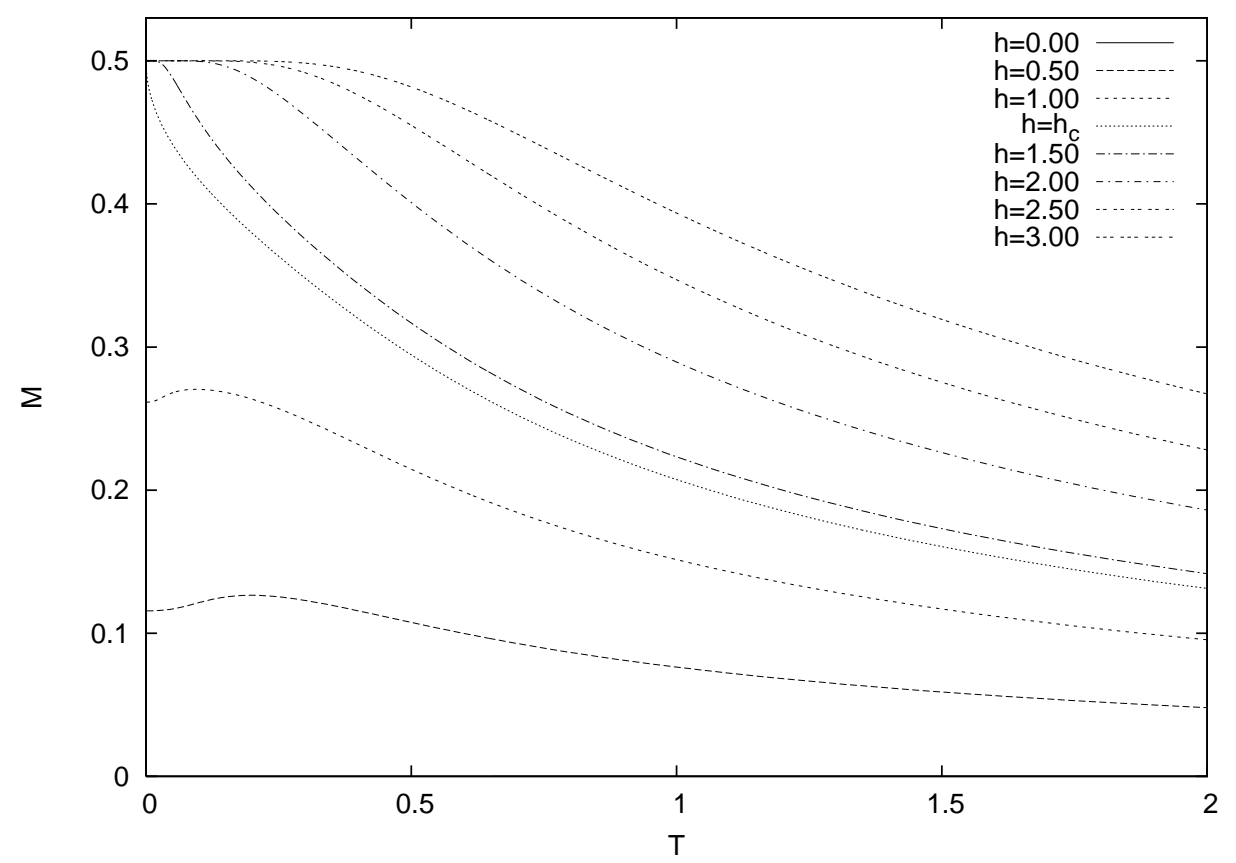

(a) Magnetisation vs. temperature.

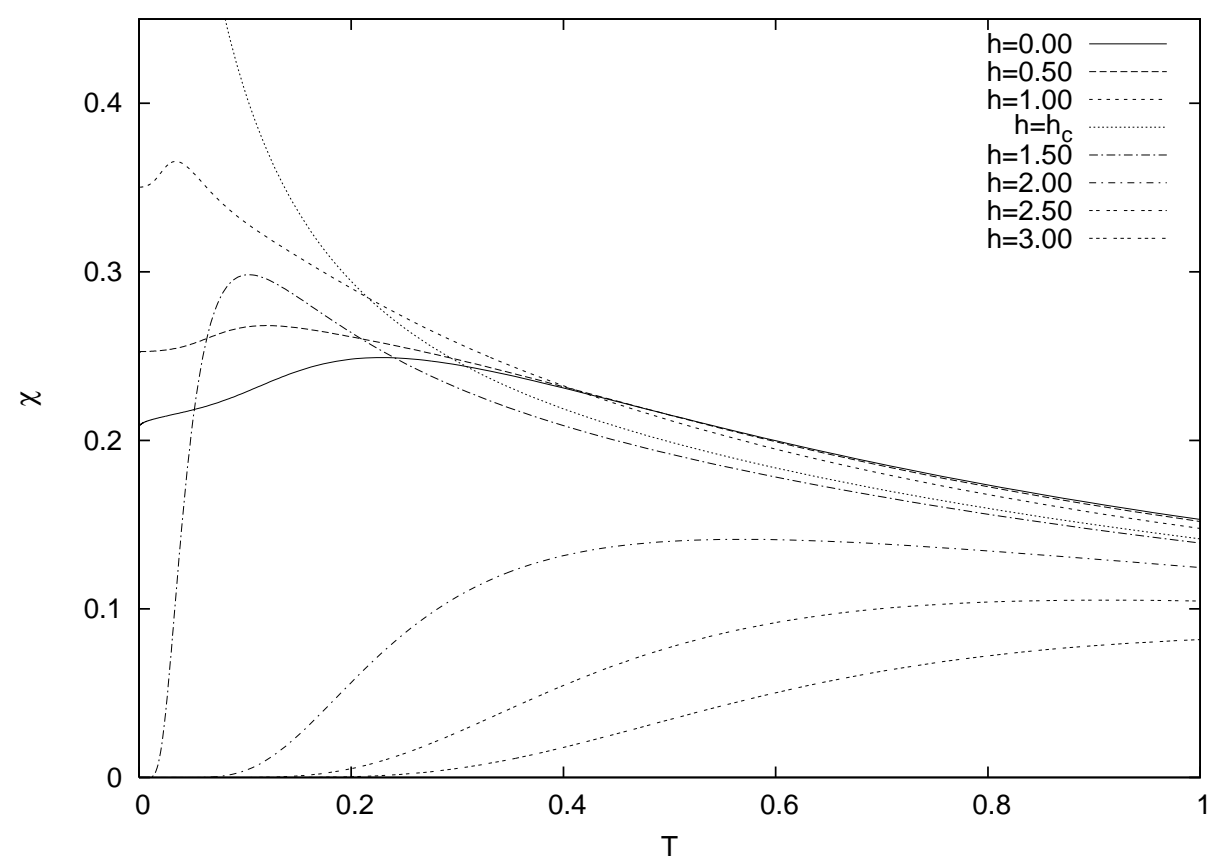

(b) Magnetic susceptibility vs. temperature.

Figure 3: Magnetisation and magnetic susceptibility of the spin-orbital model at $g_{S}=1$, $g_{\tau}=0$ for various magnetic fields. The critical field is $h_{c}=2 \ln 2 \approx 1.39$. 


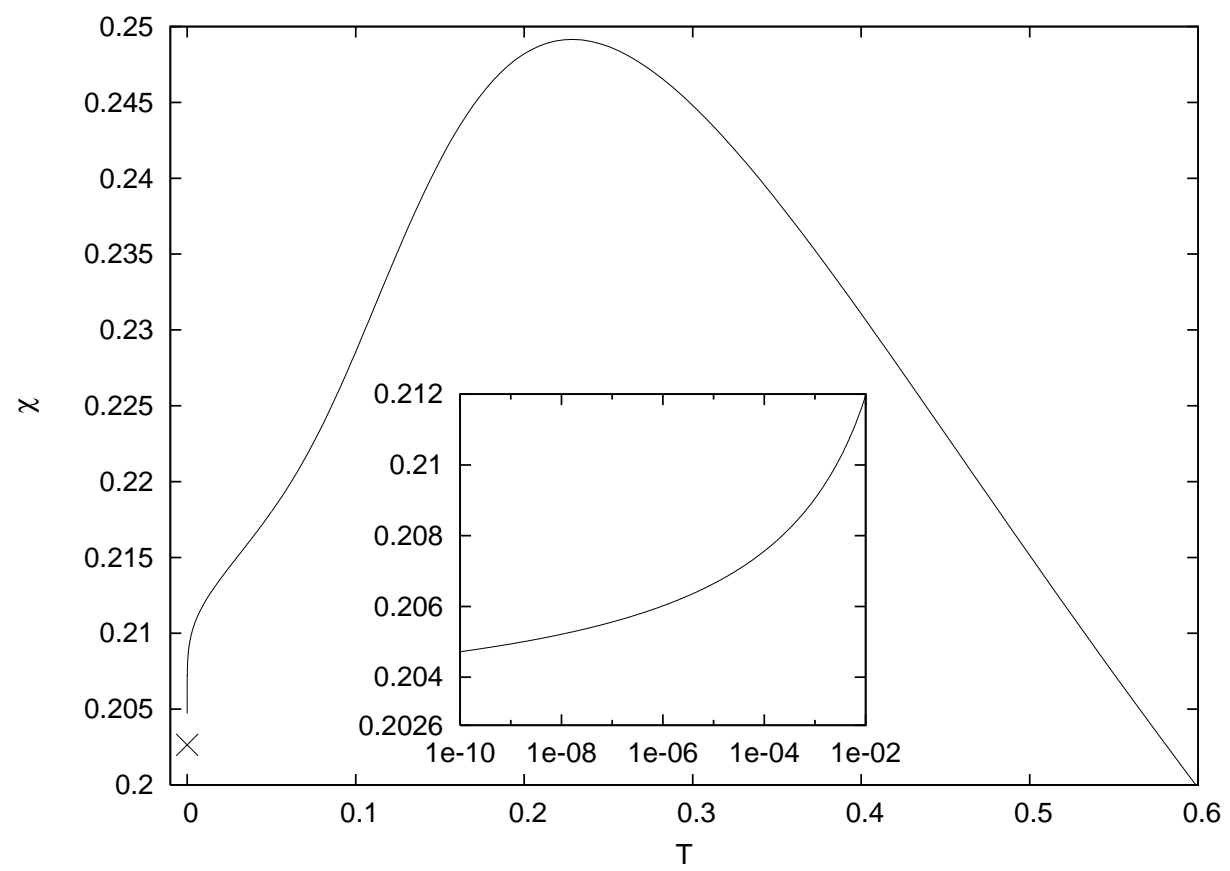

Figure 4: Magnetic susceptibility of the spin-orbital model at $h=0$ for temperatures down to $T=10^{-10}$. The cross denotes the ground-state value $\chi(0)=2 / \pi^{2} \approx 0.2026$. The inset shows the low-temperature part of the susceptibility using a logarithmic scale.

the critical field, all four states contribute to the ground state. Above, the spins are fully polarised and only the orbital degrees of freedom remain. Hence, the ground state resembles that of the spin-1/2 Heisenberg chain. This phase transition is clearly exposed by the numerical data. The low-temperature slopes both of the entropy and the specific heat increase from 2 at $h=0$ to infinity at $h=h_{c}$, whereas they are $1 / 3$ for $h>h_{c}$. Moreover, the magnetisation data show the expected saturation behaviour for $h \geq h_{c}$. Notice also, that the magnetic susceptibility diverges at the critical field. Below, the value at $T=0$ stays finite; above, it drops to zero.

The magnetic susceptibility at $h=0$ is particularly interesting as it is expected to show a characteristic singular behaviour at $T=0$ due to logarithmic corrections. Indeed, this is confirmed by our results for the low-temperature susceptibility, see Figure 4. Even for the lowest plotted temperature, $T=10^{-10}$, the susceptibility is still well above the ground-state value $\chi(0)=2 / \pi^{2}$. For the spin- $1 / 2$ Heisenberg model, these corrections have already been treated in detail [55, 56, 57, 58]; similar results for $s l(q)$-symmetric US models are known [14, 33].

We also consider the spin orbital-model at $g_{S}=1$ and $g_{\tau}=2$, which corresponds to the spin-3/2 interpretation. Here we have all three possible types of phase transitions. Numerical results for this case showing the rich resulting structure are plotted in Figure 5 on the following page and Figure 6 on page 21. Again, we observe that the low-temperature susceptibility shows characteristic singular behaviour at $h=0$ and di- 


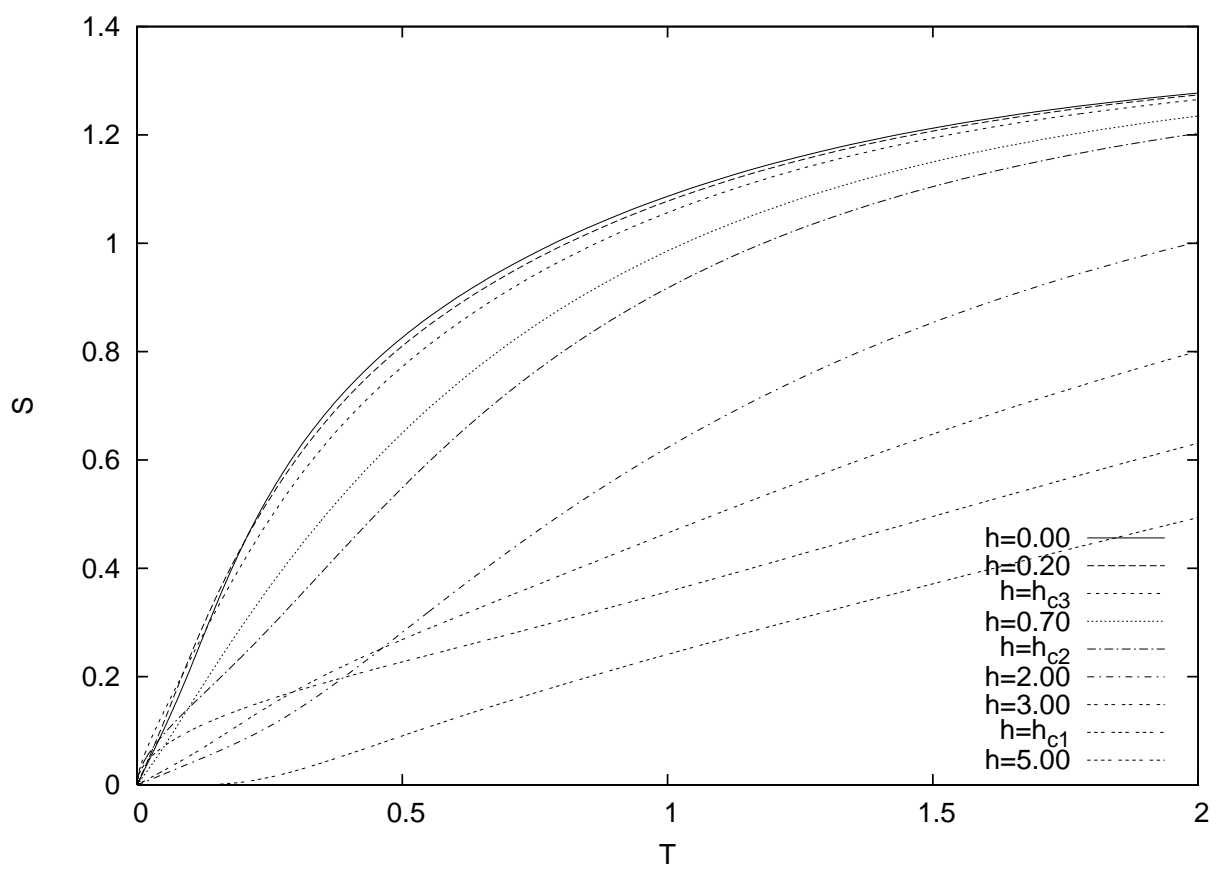

(a) Entropy vs. temperature.

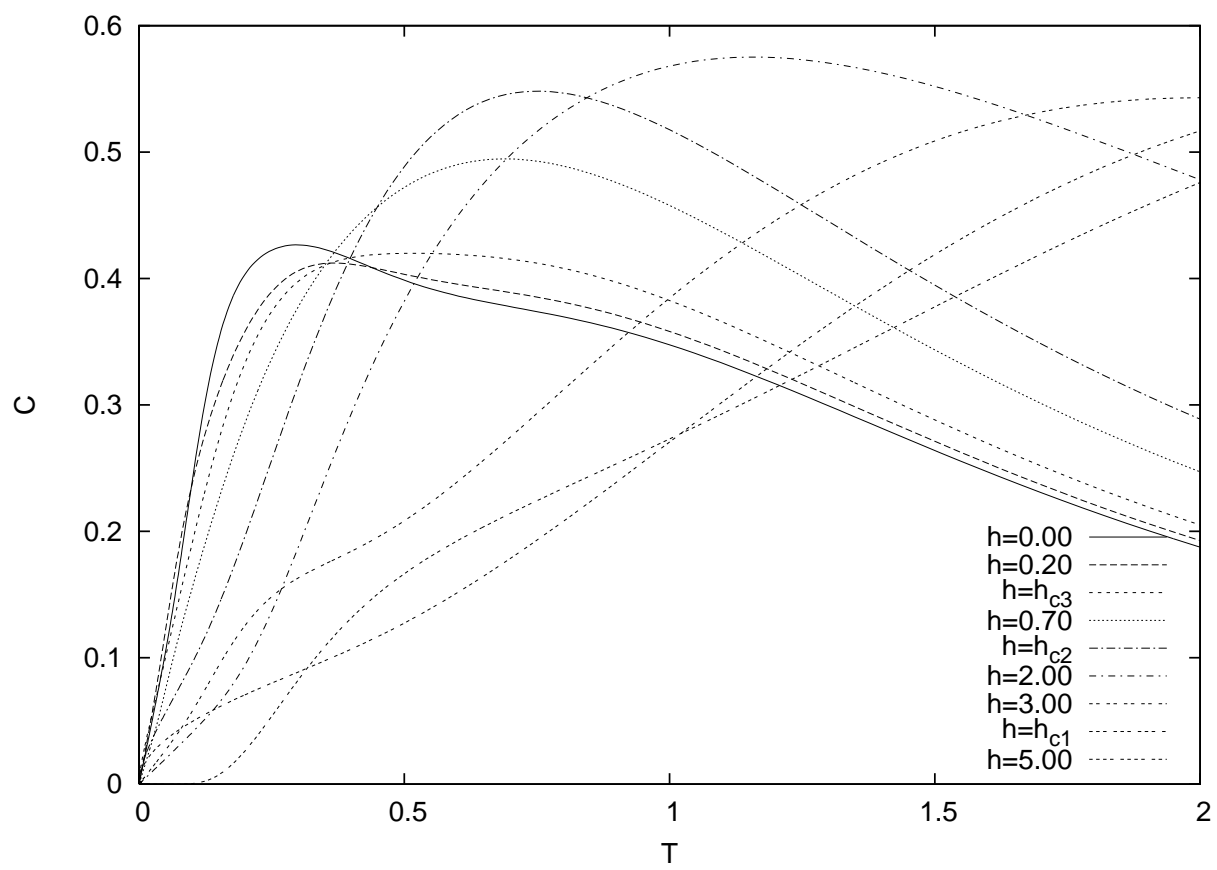

(b) Specific heat vs. temperature.

Figure 5: Entropy and specific heat of the spin-orbital model at $g_{S}=1, g_{\tau}=2$ for various magnetic fields. The critical fields are $h_{c 1}=4, h_{c 2} \approx 0.941$ and $h_{c 3} \approx 0.370$. Note the numbering of the critical fields as discussed on page 22 . 


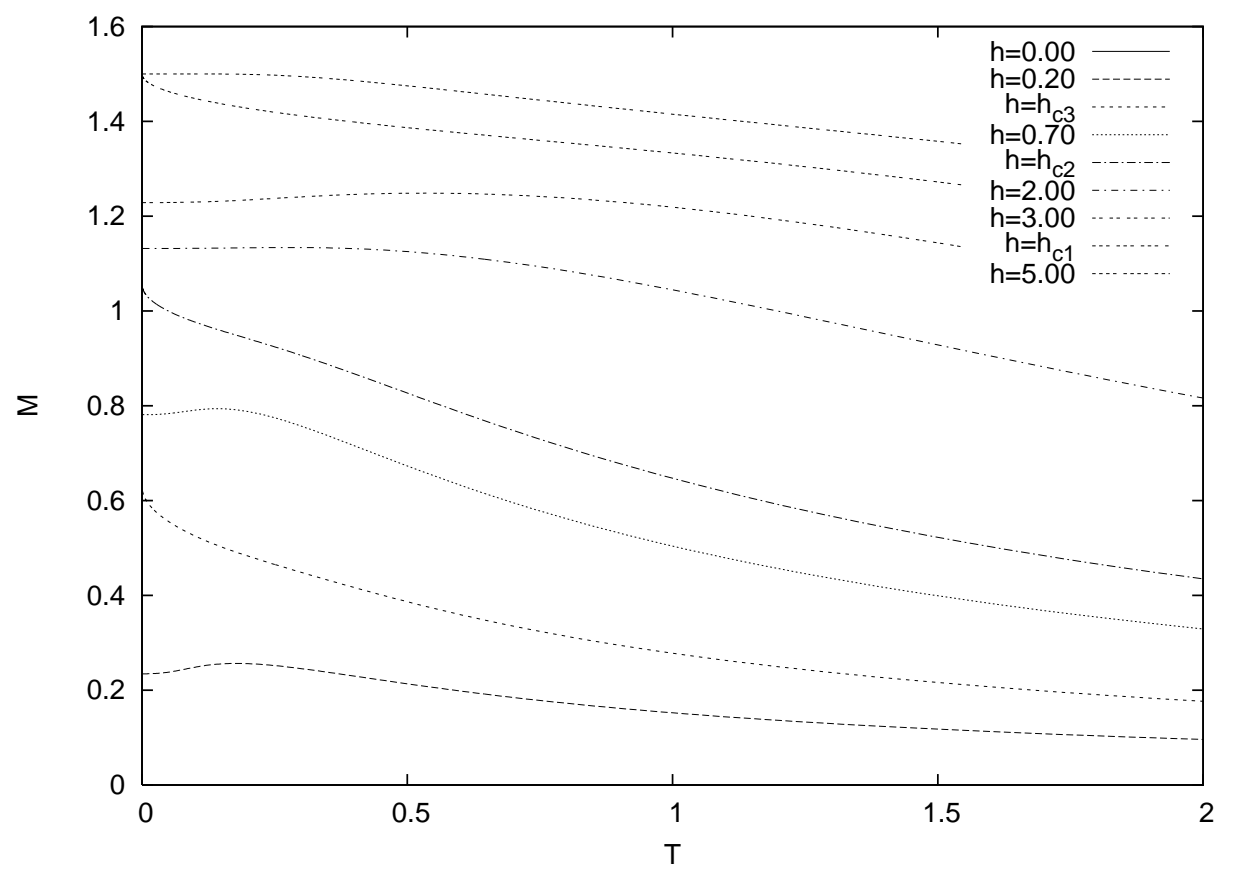

(a) Magnetisation vs. temperature.

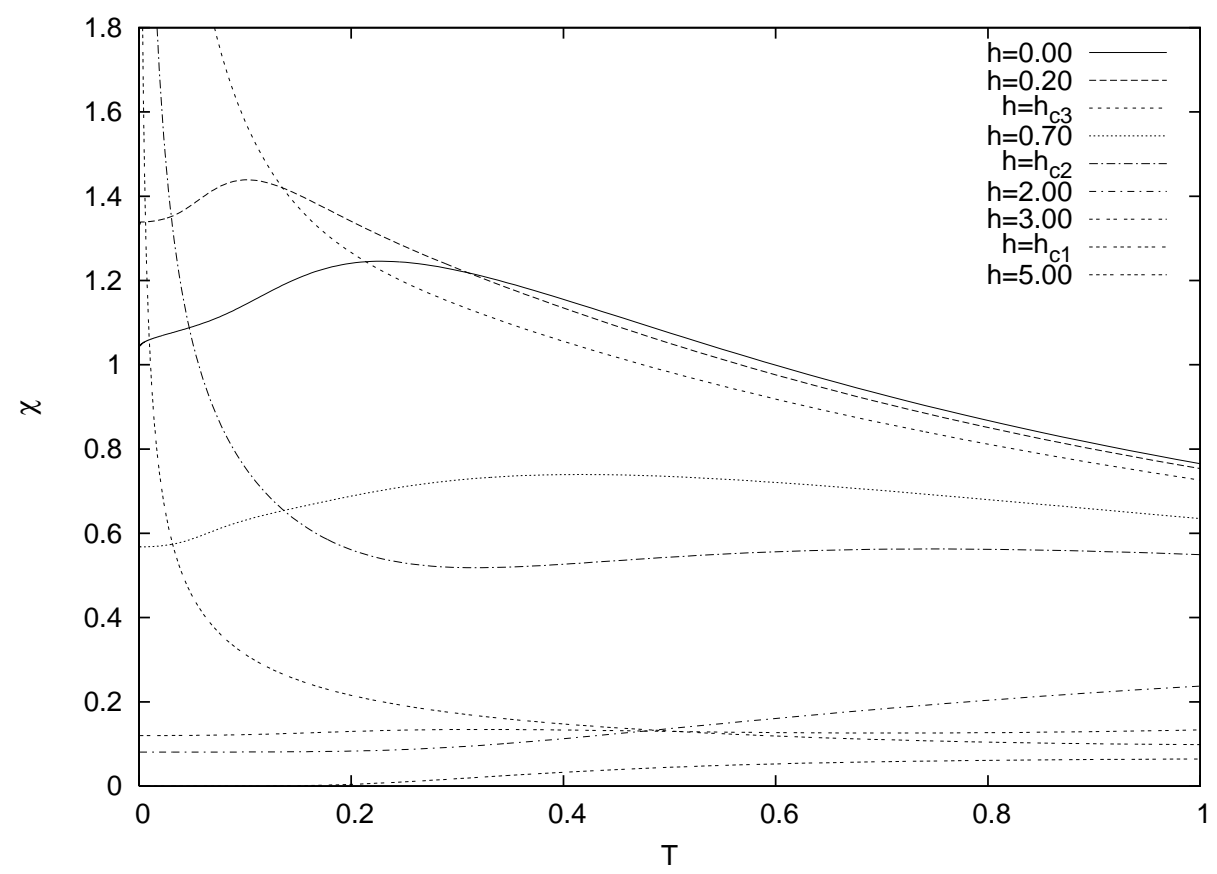

(b) Magnetic susceptibility vs. temperature.

Figure 6: Magnetisation and magnetic susceptibility of the spin-orbital model at $g_{S}=1$, $g_{\tau}=2$ for various magnetic fields. The critical fields are $h_{c 1}=4, h_{c 2} \approx 0.941$ and $h_{c 3} \approx 0.370$. Note the numbering of the critical fields as discussed on the next page. 


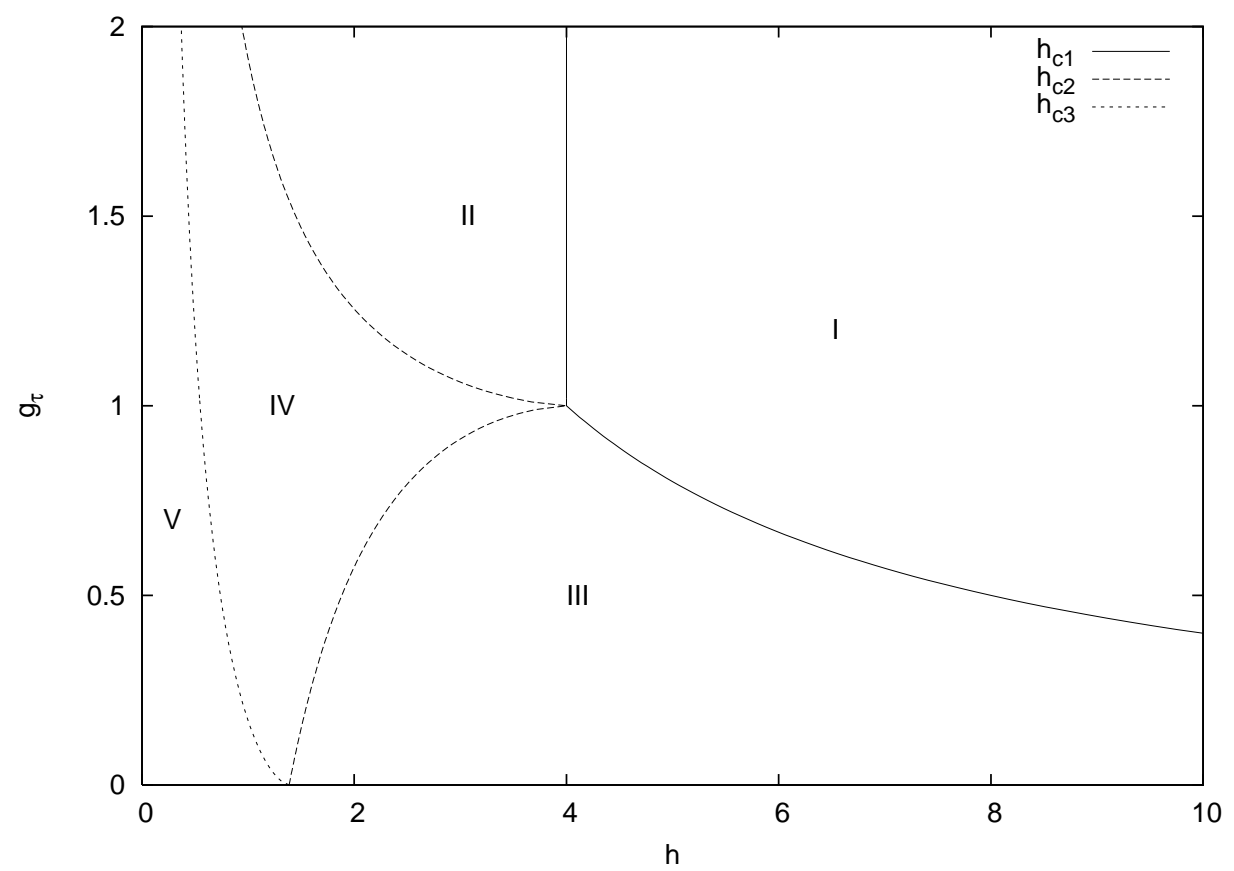

Figure 7: Phase diagram of the spin-orbital model for magnetic field $h$ and Landé factor $g_{\tau}$, where $g_{S}=1$ is held constant. There exist five different phases (I-V), for details see the text on the current page.

verges at the critical fields. The highest critical field is at $h_{c 1}=4$, the other two have to be calculated numerically as only the lower bounds $h_{c 2}>4 \ln (2) / 3 \approx 0.924$ and $h_{c 3}>\pi /(2 \sqrt{3})-\ln (3) / 2 \approx 0.358$ are known explicitly. We find the remaining critical fields to be $h_{c 2} \approx 0.941$ and $h_{c 3} \approx 0.370$. Note the numbering of the critical fields, where at each field $h_{c j}$ the number of involved degrees of freedom changes from $j$ to $j+1$. The advantage of this scheme is that the critical fields $h_{c 1}$ and $h_{c 2}$ also appear in the spin-1 interpretation of the $s l(3)$-symmetric US model [33], while only $h_{c 1}$ remains in the spin- $1 / 2$ Heisenberg model.

Figure 7 shows the complete phase diagram of the spin-orbital model depending on the magnetic field $h$ and the orbital Landé factor $g_{\tau}$, while the Landé factor of the spins is set to $g_{S}=1$. Obviously, there exist five different phases. Above $h_{c 1}$ (I), all spins and orbitals are fully polarised. Between $h_{c 1}$ and $h_{c 2}$, we have to distinguish the regions $g_{\tau}>1$ (II) and $g_{\tau}<1$ (III). In the first region, the orbitals are fully polarised, while the spins are only partially aligned. In the latter case, it is the other way around. For $g_{\tau}=1$, we have a direct transition from phase I to phase IV, because $h_{c 1}=h_{c 2}$. For a magnetic field below $h_{c 2}$, but above $h_{c 3}$ (IV), the spins and orbitals are both partially polarised, while the state $\left|\downarrow_{S} \downarrow_{\tau}\right\rangle$ is still completely suppressed. For $h<h_{c 3}(\mathrm{~V})$, all possible spin configurations contribute to the ground state. Notice that $h_{c 1}$ tends to infinity for $g_{\tau} \rightarrow 0$. As we have seen before, only one phase transition survives for $g_{\tau}=0$, where the magnetic field couples only to the spins. The phase diagram presented here is 
qualitatively in perfect agreement with [43], where a finite system of 200 sites has been used for the calculation.

\section{Summary and outlook}

We presented a set of suitable auxiliary functions that allowed us to derive a finite set of NLIE for the thermodynamics of the $s l(4)$-symmetric one-dimensional US model. These NLIE are well posed for an efficient numerical treatment using the fast Fourier transform as they are of convolution type. Moreover, they are valid for the complete temperature range and arbitrary chemical potentials. We have provided analytical results for several limiting cases of our NLIE. Thus, we recovered the previously known NLIE of the $\operatorname{sl}(3)$ case and the linearised integral equations for $T=0$. Both provide further support for the validity of the NLIE. Using the latter limit, we were able to derive all critical fields. We also gave some numerical results for the $S U(4)$ spin-orbital model as an example for the application of the $s l(4)$-symmetric US model, achieving high accuracy even at low temperatures.

This is the first time that NLIE of this type were derived for the $s l(q)$-symmetric US model with $q>3$. Unfortunately, up to now there is no general scheme to construct the needed auxiliary functions for the case $q \geq 5$. The functions presented here were basically found by trial and error. Even at the level of the NLIE, it proves difficult to generalise the structure of the somewhat complicated kernel matrix. Once the NLIE are known for some number of states $q$, there is a straightforward way to extract the NLIE of all $s l(n)$-symmetric cases with $n<q$ by freezing out one or more of the states. Going the opposite way fixes some of the structure, but does not provide enough information to deduce the entries of the kernel matrix lying on the anti-diagonal. Nevertheless, we hope to overcome these difficulties and to generalise the approach to cover the whole class of $s l(q)$-symmetric models in the near future. For that, we expect that the number of required auxiliary functions will be equal to the sum of the dimensions of all fundamental representations of $\operatorname{sl}(q)$, i.e. $2^{q}-2$. We note that a further generalisation of this type of NLIE from the fundamental representation to higher rank representations [5] should be quite straightforward. To achieve this, one has to consider slightly modified auxiliary functions, which can be used to truncate the TBA equations at an arbitrary step, in analogy to [30].

Another open question is the connection between the kernel matrix and the complete $S$-matrix of elementary excitations [59]. In the $T=0$ case, all kernel functions can be obtained from corresponding $S$-matrix entries [54], the generalisation to $T>0$ is unknown.

The treatment of graded models is a further direction of generalisation. Up to now, only the $\operatorname{sl}(2 \mid 1)$-symmetric case has been treated this way [31, 32]. Although supersymmetric models are generally more difficult, it turns out that, at least for the $s l(2 \mid 1)$ case, the corresponding NLIE are even simpler than the NLIE of the $s l(3)$ case as only three auxiliary functions are needed. The NLIE for the graded $q=4$ models, e.g. the $s l(2 \mid 2)$ case [60], also seem to be simpler than the NLIE presented here. We hope to report soon 
on details concerning this model.

\section{Acknowledgments}

The authors like to acknowledge support by the research program of the Graduiertenkolleg 1052 funded by the Deutsche Forschungsgemeinschaft.

\section{A Derivation of the NLIE}

The auxiliary functions, as defined in (23)-(25), are rational functions. Moreover, we have numerically checked that they are analytic, non-zero and have constant asymptotics (ANZC) in a strip $-1 / 2 \lesssim \Im(x) \lesssim 1 / 2$, which includes the real axis. Therefore, we are allowed to apply a Fourier transform to the logarithmic derivative of all auxiliary functions,

$$
\widehat{f}(k)=\int_{-\infty}^{\infty} \frac{\mathrm{d}}{\mathrm{d} x}[\ln f(x)] \mathrm{e}^{-\mathrm{i} k x} \frac{\mathrm{d} x}{2 \pi} .
$$

In the cases $k<0$ and $k>0$, we can close the integration path to a contour above and below the real axis, respectively. Hence, it is important to know in which of these regions the first-order poles of the logarithmic derivatives are located and how they can be further classified.

Let us first introduce some notation. We define the constants

$$
\begin{aligned}
a^{(1)}= & \mathrm{e}^{\beta \mu_{1}}+\mathrm{e}^{\beta \mu_{2}}+\mathrm{e}^{\beta \mu_{3}}+\mathrm{e}^{\beta \mu_{4}}, \\
a^{(2)}= & \mathrm{e}^{\beta\left(\mu_{1}+\mu_{2}\right)}+\mathrm{e}^{\beta\left(\mu_{1}+\mu_{3}\right)}+\mathrm{e}^{\beta\left(\mu_{1}+\mu_{4}\right)} \\
& \quad+\mathrm{e}^{\beta\left(\mu_{2}+\mu_{3}\right)}+\mathrm{e}^{\beta\left(\mu_{2}+\mu_{4}\right)}+\mathrm{e}^{\beta\left(\mu_{3}+\mu_{4}\right)}, \\
a^{(3)}= & \mathrm{e}^{\beta\left(\mu_{1}+\mu_{2}+\mu_{3}\right)}+\mathrm{e}^{\beta\left(\mu_{1}+\mu_{2}+\mu_{4}\right)}+\mathrm{e}^{\beta\left(\mu_{1}+\mu_{3}+\mu_{4}\right)}+\mathrm{e}^{\beta\left(\mu_{2}+\mu_{3}+\mu_{4}\right)}, \\
\varphi_{1}= & \mathrm{e}^{\beta \mu_{1}}+\mathrm{e}^{\beta \mu_{2}}, \quad \varphi_{2}=\mathrm{e}^{\beta \mu_{2}}+\mathrm{e}^{\beta \mu_{3}}, \quad \varphi_{3}=\mathrm{e}^{\beta \mu_{3}}+\mathrm{e}^{\beta \mu_{4}}, \\
\chi_{1}^{(1)}= & \mathrm{e}^{\beta \mu_{1}}+\mathrm{e}^{\beta \mu_{2}}+\mathrm{e}^{\beta \mu_{3}}, \quad \chi_{2}^{(1)}=\mathrm{e}^{\beta \mu_{2}}+\mathrm{e}^{\beta \mu_{3}}+\mathrm{e}^{\beta \mu_{4}}, \\
\chi_{1}^{(2)}= & \mathrm{e}^{\beta\left(\mu_{1}+\mu_{2}\right)}+\mathrm{e}^{\beta\left(\mu_{1}+\mu_{3}\right)}+\mathrm{e}^{\beta\left(\mu_{1}+\mu_{4}\right)}+\mathrm{e}^{\beta\left(\mu_{2}+\mu_{3}\right)}+\mathrm{e}^{\beta\left(\mu_{2}+\mu_{4}\right)}, \\
\chi_{2}^{(2)}= & \mathrm{e}^{\beta\left(\mu_{1}+\mu_{3}\right)}+\mathrm{e}^{\beta\left(\mu_{1}+\mu_{4}\right)}+\mathrm{e}^{\beta\left(\mu_{2}+\mu_{3}\right)}+\mathrm{e}^{\beta\left(\mu_{2}+\mu_{4}\right)}+\mathrm{e}^{\beta\left(\mu_{3}+\mu_{4}\right)}, \\
\chi_{1}^{(3)}= & \mathrm{e}^{\beta\left(\mu_{1}+\mu_{2}\right)}+\mathrm{e}^{\beta\left(\mu_{1}+\mu_{3}\right)}+\mathrm{e}^{\beta\left(\mu_{2}+\mu_{3}\right)}, \\
\chi_{2}^{(3)}= & \mathrm{e}^{\beta\left(\mu_{2}+\mu_{3}\right)}+\mathrm{e}^{\beta\left(\mu_{2}+\mu_{4}\right)}+\mathrm{e}^{\beta\left(\mu_{3}+\mu_{4}\right)} .
\end{aligned}
$$

For the eigenvalues (22), we find the factorisation

$$
\begin{aligned}
& \Lambda^{(1)}(x)=\widetilde{\Lambda}^{(1)}(x) \cdot a^{(1)} \\
& \Lambda^{(2)}(x)=\phi_{-}(x+\mathrm{i} / 2) \phi_{+}(x-\mathrm{i} / 2) \widetilde{\Lambda}^{(2)}(x) \cdot a^{(2)}, \\
& \Lambda^{(3)}(x)=\phi_{-}(v) \phi_{-}(v+\mathrm{i}) \phi_{+}(v-\mathrm{i}) \phi_{+}(v) \widetilde{\Lambda}^{(3)}(x) \cdot a^{(3)},
\end{aligned}
$$


where all $\widetilde{\Lambda}^{(n)}(x)$ are polynomials of degree $N$ with the highest coefficients being one. For the other terms in the auxiliary functions, which are generated by sums of certain Young tableaux, we also find that several of their potential poles vanish due to the BA equations (18). We can therefore write

$$
\begin{aligned}
& 1+\left.2\right|_{v=x}=\frac{\phi_{+}(x) q_{1}^{(h)}(x)}{q_{2}(x)} \cdot \varphi_{1} \\
& 2+\left.3\right|_{v=x}=\frac{\phi_{-}(x) \phi_{+}(x) q_{2}^{(h)}(x)}{q_{1}(x) q_{3}(x)} \cdot \varphi_{2}, \\
& 3+\left.4\right|_{v=x}=\frac{\phi_{-}(x) q_{3}^{(h)}(x)}{q_{2}(x)} \cdot \varphi_{3} \text {, } \\
& 1+2+\left.3\right|_{v=x}=\frac{\phi_{+}(x) X_{1}^{(1)}(x)}{q_{3}(x)} \cdot \chi_{1}^{(1)}, \\
& 2+3+\left.4\right|_{v=x}=\frac{\phi_{-}(x) X_{2}^{(1)}(x)}{q_{1}(x)} \cdot \chi_{2}^{(1)}, \\
& \frac{1}{2}+\frac{1}{3}+\frac{1}{4}+\frac{2}{3}+\left.\frac{2}{4}\right|_{v=x}=\frac{\phi_{-}\left(x+\frac{\mathrm{i}}{2}\right) \phi_{+}\left(x-\frac{\mathrm{i}}{2}\right) X_{1}^{(2)}(x)}{q_{2}\left(x-\frac{\mathrm{i}}{2}\right)} \cdot \chi_{1}^{(2)} \text {, } \\
& \begin{array}{|l|}
\frac{1}{3} \\
\hline
\end{array}+\frac{1}{4}+\frac{2}{3}+\frac{2}{4}+\left.\frac{3}{4}\right|_{v=x}=\frac{\phi_{-}\left(x+\frac{\mathrm{i}}{2}\right) \phi_{+}\left(x-\frac{\mathrm{i}}{2}\right) X_{2}^{(2)}(x)}{q_{2}\left(x+\frac{\mathrm{i}}{2}\right)} \cdot \chi_{2}^{(2)} \text {, } \\
& \frac{1}{2}+\frac{1}{3}+\left.\frac{2}{3}\right|_{v=x}=\frac{\phi_{-}\left(x+\frac{\mathrm{i}}{2}\right) \phi_{+}\left(x-\frac{\mathrm{i}}{2}\right) \phi_{+}\left(x+\frac{\mathrm{i}}{2}\right) X_{1}^{(3)}(x)}{q_{3}\left(x+\frac{\mathrm{i}}{2}\right)} \cdot \chi_{1}^{(3)} \text {, } \\
& \begin{array}{l}
2 \\
3
\end{array}+\frac{2}{4}+\left.\frac{3}{4}\right|_{v=x}=\frac{\phi_{-}\left(x+\frac{\mathrm{i}}{2}\right) \phi_{+}\left(x-\frac{\mathrm{i}}{2}\right) \phi_{-}\left(x-\frac{\mathrm{i}}{2}\right) X_{2}^{(3)}(x)}{q_{1}\left(x-\frac{\mathrm{i}}{2}\right)} \cdot \chi_{2}^{(3)} \text {. }
\end{aligned}
$$

All functions $q_{j}^{(h)}(x)$ and $X_{j}^{(n)}(x)$ are polynomials of degree $N$, with the exception of $X_{1}^{(2)}(x)$ and $X_{2}^{(2)}(x)$ being of degree $3 N / 2$, and with a highest coefficient of one. We note that the roots of the functions $q_{j}^{(h)}(x)$ provide additional solutions to the corresponding BA equations (18) and are called the hole-type solutions.

The roots of the polynomials can be obtained from numerical solutions of the BA equations at finite $N$. We find that, in the complex plane, all roots are located on slightly curved lines close to horizontal axes at certain heights. Each curve contains $N / 2$ many roots. The corresponding height values for each polynomial are given in the following table:

$$
\begin{array}{rlrl}
q_{j}(x) & : 0 & \widetilde{\Lambda}^{(j)}(x): \pm(j+1) / 2 \\
q_{j}^{(h)}(x): \pm 1 & X_{j}^{(1)}(x): \pm 1 \\
X_{1}^{(2)}(x):+1 / 2, \pm 3 / 2 & X_{2}^{(2)}(x):-1 / 2, \pm 3 / 2 \\
X_{j}^{(3)}(x): \pm 3 / 2 &
\end{array}
$$


We like to stress that the deviations from these axes remain small even for large $N$.

Next, we write the auxiliary functions $b_{j}^{(n)}(x)$ in a factorised form, from which one can easily read off the locations of all roots and poles,

$$
\begin{aligned}
& b_{1}^{(1)}(x)=\frac{\phi_{-}\left(x-\frac{\mathrm{i}}{2}\right) \phi_{+}\left(x+\frac{\mathrm{i}}{2}\right) q_{1}\left(x+\frac{3}{2} \mathrm{i}\right)}{\phi_{-}\left(x+\frac{\mathrm{i}}{2}\right) X_{2}^{(1)}\left(x+\frac{\mathrm{i}}{2}\right)} \cdot \frac{\mathrm{e}^{\beta \mu_{1}}}{\chi_{2}^{(1)}}, \\
& b_{2}^{(1)}(x)=\frac{\phi_{-}\left(x-\frac{\mathrm{i}}{2}\right) \phi_{+}\left(x+\frac{\mathrm{i}}{2}\right) q_{2}\left(x+\frac{3}{2} \mathrm{i}\right) X_{2}^{(3)}(x)}{q_{1}\left(x+\frac{\mathrm{i}}{2}\right) q_{3}^{(h)}\left(x+\frac{\mathrm{i}}{2}\right) \widetilde{\Lambda}^{(2)}(x)} \cdot \frac{\mathrm{e}^{\beta \mu_{2}} \chi_{2}^{(3)}}{\varphi_{3} a^{(2)}}, \\
& b_{3}^{(1)}(x)=\frac{\phi_{-}\left(x-\frac{\mathrm{i}}{2}\right) \phi_{+}\left(x+\frac{\mathrm{i}}{2}\right) q_{2}\left(x-\frac{3}{2} \mathrm{i}\right) q_{3}\left(x+\frac{3}{2} \mathrm{i}\right)}{q_{3}\left(x-\frac{\mathrm{i}}{2}\right) X_{2}^{(2)}(x)} \cdot \frac{\mathrm{e}^{2 \beta \mu_{3}}}{\chi_{2}^{(2)}}, \\
& b_{4}^{(1)}(x)=\frac{\phi_{-}\left(x-\frac{\mathrm{i}}{2}\right) \phi_{+}\left(x+\frac{\mathrm{i}}{2}\right) q_{3}\left(x-\frac{3}{2} \mathrm{i}\right)}{\phi_{+}\left(x-\frac{\mathrm{i}}{2}\right) X_{1}^{(1)}\left(x-\frac{\mathrm{i}}{2}\right)} \cdot \frac{\mathrm{e}^{\beta \mu_{4}}}{\chi_{1}^{(1)}}, \\
& b_{1}^{(2)}(x)=\frac{\phi_{-}(x-\mathrm{i}) \phi_{+}(x+\mathrm{i}) q_{2}(x+2 \mathrm{i})}{X_{2}^{(2)}\left(x+\frac{\mathrm{i}}{2}\right)} \cdot \frac{\mathrm{e}^{\beta\left(\mu_{1}+\mu_{2}\right)}}{\chi_{2}^{(2)}}, \\
& b_{2}^{(2)}(x)=\frac{\phi_{-}(x-\mathrm{i}) \phi_{+}(x+\mathrm{i}) q_{1}(x+\mathrm{i}) q_{2}(x-\mathrm{i}) q_{3}(x+2 \mathrm{i})}{q_{2}(x+\mathrm{i}) X_{1}^{(1)}(x) X_{2}^{(3)}\left(x+\frac{\mathrm{i}}{2}\right)} \cdot \frac{\mathrm{e}^{\beta\left(\mu_{1}+2 \mu_{3}\right)}}{\chi_{1}^{(1)} \chi_{2}^{(3)}}, \\
& b_{3}^{(2)}(x)=\frac{\phi_{-}(x-\mathrm{i}) \phi_{+}(x+\mathrm{i}) q_{1}(x+\mathrm{i}) q_{3}(x-\mathrm{i})}{q_{2}^{(h)}(x) \widetilde{\Lambda}^{(1)}(x)} \cdot \frac{\mathrm{e}^{\beta\left(\mu_{1}+\mu_{4}\right)}}{\varphi_{2} a^{(1)}}, \\
& b_{4}^{(2)}(x)=\frac{\phi_{-}(x-\mathrm{i}) \phi_{+}(x+\mathrm{i}) q_{1}(x-2 \mathrm{i}) q_{3}(x+2 \mathrm{i})}{q_{2}^{(h)}(x) \widetilde{\Lambda}^{(3)}(x)} \cdot \frac{\mathrm{e}^{2 \beta\left(\mu_{2}+\mu_{3}\right)}}{\varphi_{2} a^{(3)}}, \\
& b_{5}^{(2)}(x)=\frac{\phi_{-}(x-\mathrm{i}) \phi_{+}(x+\mathrm{i}) q_{1}(x-2 \mathrm{i}) q_{2}(x+\mathrm{i}) q_{3}(x-\mathrm{i})}{q_{2}(x-\mathrm{i}) X_{2}^{(1)}(x) X_{1}^{(3)}\left(x-\frac{\mathrm{i}}{2}\right)} \cdot \frac{\mathrm{e}^{\beta\left(2 \mu_{2}+\mu_{4}\right)}}{\chi_{2}^{(1)} \chi_{1}^{(3)}}, \\
& b_{6}^{(2)}(x)=\frac{\phi_{-}(x-\mathrm{i}) \phi_{+}(x+\mathrm{i}) q_{2}(x-2 \mathrm{i})}{X_{1}^{(2)}\left(x-\frac{\mathrm{i}}{2}\right)} \cdot \frac{\mathrm{e}^{\beta\left(\mu_{3}+\mu_{4}\right)}}{\chi_{1}^{(2)}}, \\
& b_{1}^{(3)}(x)=\frac{\phi_{-}\left(x-\frac{3}{2} \mathrm{i}\right) \phi_{+}\left(x+\frac{3}{2} \mathrm{i}\right) q_{3}\left(x+\frac{5}{2} \mathrm{i}\right)}{\phi_{+}\left(x+\frac{5}{2} \mathrm{i}\right) X_{1}^{(3)}(x)} \cdot \frac{\mathrm{e}^{\beta\left(\mu_{1}+\mu_{2}+\mu_{3}\right)}}{\mathrm{e}^{\beta \mu_{4}} \chi_{1}^{(3)}}, \\
& b_{2}^{(3)}(x)=\frac{\phi_{-}\left(x-\frac{3}{2} \mathrm{i}\right) \phi_{+}\left(x+\frac{3}{2} \mathrm{i}\right) q_{2}\left(x+\frac{3}{2} \mathrm{i}\right) q_{3}\left(x-\frac{\mathrm{i}}{2}\right)}{q_{3}\left(x+\frac{3}{2} \mathrm{i}\right) X_{1}^{(2)}(x)} \cdot \frac{\mathrm{e}^{\beta\left(\mu_{1}+\mu_{2}+\mu_{4}\right)}}{\mathrm{e}^{\beta \mu_{3}} \chi_{1}^{(2)}}, \\
& b_{3}^{(3)}(x)=\frac{\phi_{-}\left(x-\frac{3}{2} \mathrm{i}\right) \phi_{+}\left(x+\frac{3}{2} \mathrm{i}\right) q_{2}\left(x-\frac{3}{2} \mathrm{i}\right) X_{2}^{(1)}\left(x+\frac{\mathrm{i}}{2}\right)}{q_{1}\left(x-\frac{3}{2} \mathrm{i}\right) q_{3}^{(h)}\left(x+\frac{\mathrm{i}}{2}\right) \widetilde{\Lambda}^{(2)}(x)} \cdot \frac{\mathrm{e}^{\beta\left(\mu_{1}+\mu_{3}+\mu_{4}\right)} \chi_{2}^{(1)}}{\mathrm{e}^{\beta \mu_{2}} \varphi_{3} a^{(2)}}, \\
& b_{4}^{(3)}(x)=\frac{\phi_{-}\left(x-\frac{3}{2} \mathrm{i}\right) \phi_{+}\left(x+\frac{3}{2} \mathrm{i}\right) q_{1}\left(x-\frac{5}{2} \mathrm{i}\right)}{\phi_{-}\left(x-\frac{5}{2} \mathrm{i}\right) X_{2}^{(3)}(x)} \cdot \frac{\mathrm{e}^{\beta\left(\mu_{2}+\mu_{3}+\mu_{4}\right)}}{\mathrm{e}^{\beta \mu_{1}} \chi_{2}^{(3)}} \text {. }
\end{aligned}
$$


The uppercase auxiliary functions $B_{j}^{(n)}(x)$ factorise in an analogous way,

$$
\begin{aligned}
& B_{1}^{(1)}(x)=\frac{q_{1}\left(x+\frac{\mathrm{i}}{2}\right) \widetilde{\Lambda}^{(1)}\left(x+\frac{\mathrm{i}}{2}\right)}{\phi_{-}\left(x+\frac{\mathrm{i}}{2}\right) X_{2}^{(1)}\left(x+\frac{\mathrm{i}}{2}\right)} \cdot \frac{a^{(1)}}{\chi_{2}^{(1)}}, \\
& B_{2}^{(1)}(x)=\frac{X_{2}^{(1)}\left(x+\frac{\mathrm{i}}{2}\right) X_{2}^{(2)}(x)}{q_{1}\left(x+\frac{\mathrm{i}}{2}\right) q_{3}^{(h)}\left(x+\frac{\mathrm{i}}{2}\right) \widetilde{\Lambda}^{(2)}(x)} \cdot \frac{\chi_{2}^{(1)} \chi_{2}^{(2)}}{\varphi_{3} a^{(2)}}, \\
& B_{3}^{(1)}(x)=\frac{q_{3}^{(h)}\left(x+\frac{\mathrm{i}}{2}\right) X_{1}^{(1)}\left(x-\frac{\mathrm{i}}{2}\right)}{q_{3}\left(x-\frac{\mathrm{i}}{2}\right) X_{2}^{(2)}(x)} \cdot \frac{\varphi_{3} \chi_{1}^{(1)}}{\chi_{2}^{(2)}}, \\
& B_{4}^{(1)}(x)=\frac{q_{3}\left(x-\frac{\mathrm{i}}{2}\right) \widetilde{\Lambda}^{(1)}\left(x-\frac{\mathrm{i}}{2}\right)}{\phi_{+}\left(x-\frac{\mathrm{i}}{2}\right) X_{1}^{(1)}\left(x-\frac{\mathrm{i}}{2}\right)} \cdot \frac{a^{(1)}}{\chi_{1}^{(1)}}, \\
& B_{1}^{(2)}(x)=\frac{q_{2}(x+\mathrm{i}) \widetilde{\Lambda}^{(2)}\left(x+\frac{\mathrm{i}}{2}\right)}{X_{2}^{(2)}\left(x+\frac{\mathrm{i}}{2}\right)} \cdot \frac{a^{(2)}}{\chi_{2}^{(2)}}, \\
& B_{2}^{(2)}(x)=\frac{q_{2}^{(h)}(x) X_{2}^{(2)}\left(x+\frac{\mathrm{i}}{2}\right)}{q_{2}(x+\mathrm{i}) X_{1}^{(1)}(x) X_{2}^{(3)}\left(x+\frac{\mathrm{i}}{2}\right)} \cdot \frac{\varphi_{2} \chi_{2}^{(2)}}{\chi_{1}^{(1)} \chi_{2}^{(3)}}, \\
& B_{3}^{(2)}(x)=\frac{X_{1}^{(1)}(x) X_{2}^{(1)}(x)}{q_{2}^{(h)}(x) \widetilde{\Lambda}^{(1)}(x)} \cdot \frac{\chi_{1}^{(1)} \chi_{2}^{(1)}}{\varphi_{2} a^{(1)}}, \\
& B_{4}^{(2)}(x)=\frac{X_{1}^{(3)}\left(x-\frac{\mathrm{i}}{2}\right) X_{2}^{(3)}\left(x+\frac{\mathrm{i}}{2}\right)}{q_{2}^{(h)}(x) \widetilde{\Lambda}^{(3)}(x)} \cdot \frac{\chi_{1}^{(3)} \chi_{2}^{(3)}}{\varphi_{2} a^{(3)}}, \\
& B_{5}^{(2)}(x)=\frac{q_{2}^{(h)}(x) X_{1}^{(2)}\left(x-\frac{\mathrm{i}}{2}\right)}{q_{2}(x-\mathrm{i}) X_{2}^{(1)}(x) X_{1}^{(3)}\left(x-\frac{\mathrm{i}}{2}\right)} \cdot \frac{\varphi_{2} \chi_{1}^{(2)}}{\chi_{2}^{(1)} \chi_{1}^{(3)}}, \\
& B_{6}^{(2)}(x)=\frac{q_{2}(x-\mathrm{i}) \widetilde{\Lambda}^{(2)}\left(x-\frac{\mathrm{i}}{2}\right)}{X_{1}^{(2)}\left(x-\frac{\mathrm{i}}{2}\right)} \cdot \frac{a^{(2)}}{\chi_{1}^{(2)}}, \\
& B_{1}^{(3)}(x)=\frac{q_{3}\left(x+\frac{3}{2} \mathrm{i}\right) \widetilde{\Lambda}^{(3)}\left(x+\frac{\mathrm{i}}{2}\right)}{\phi_{+}\left(x+\frac{5}{2} \mathrm{i}\right) X_{1}^{(3)}(x)} \cdot \frac{a^{(3)}}{\mathrm{e}^{\beta \mu_{4}} \chi_{1}^{(3)}}, \\
& B_{2}^{(3)}(x)=\frac{q_{3}^{(h)}\left(x+\frac{\mathrm{i}}{2}\right) X_{1}^{(3)}(x)}{q_{3}\left(x+\frac{3}{2} \mathrm{i}\right) X_{1}^{(2)}(x)} \cdot \frac{\varphi_{3} \chi_{1}^{(3)}}{\mathrm{e}^{\beta \mu_{3}} \chi_{1}^{(2)}}, \\
& B_{3}^{(3)}(x)=\frac{X_{1}^{(2)}(x) X_{2}^{(3)}(x)}{q_{1}\left(x-\frac{3}{2} \mathrm{i}\right) q_{3}^{(h)}\left(x+\frac{\mathrm{i}}{2}\right) \widetilde{\Lambda}^{(2)}(x)} \cdot \frac{\chi_{1}^{(2)} \chi_{2}^{(3)}}{\mathrm{e}^{\beta \mu_{2}} \varphi_{3} a^{(2)}}, \\
& B_{4}^{(3)}(x)=\frac{q_{1}\left(x-\frac{3}{2} \mathrm{i}\right) \widetilde{\Lambda}_{1}^{(3)}\left(x-\frac{\mathrm{i}}{2}\right)}{\phi_{-}\left(x-\frac{5}{2} \mathrm{i}\right) X_{2}^{(3)}(x)} \cdot \frac{a^{(3)}}{\mathrm{e}^{\beta \mu_{1}} \chi_{2}^{(3)}} .
\end{aligned}
$$

Now, we apply (68) to all auxiliary functions. For brevity, we just treat the case $k<0$ here as the calculation is completely analogous for $k>0$. In this case, we just have to deal with roots and poles, which are located above the real axis. For the functions $b_{j}^{(n)}(x)$ 
we get the result

$$
\begin{aligned}
& \widehat{b}_{1}^{(1)}(k)=\mathrm{e}^{k / 2} \widehat{\phi}_{-}(k)-\mathrm{e}^{-k / 2} \widehat{X}_{2}^{(1)}(k), \\
& \widehat{b}_{2}^{(1)}(k)=\mathrm{e}^{k / 2} \widehat{\phi}_{-}(k)+\widehat{X}_{2}^{(3)}(k)-\mathrm{e}^{-k / 2} \widehat{q}_{3}^{(h)}(k)-\widehat{\Lambda}^{(2)}(k), \\
& \widehat{b}_{3}^{(1)}(k)=\mathrm{e}^{k / 2} \widehat{\phi}_{-}(k)+\mathrm{e}^{3 k / 2} \widehat{q}_{2}(k)-\mathrm{e}^{k / 2} \widehat{q}_{3}(k)-\widehat{X}_{2}^{(2)}(k), \\
& \widehat{b}_{4}^{(1)}(k)=\mathrm{e}^{k / 2} \widehat{\phi}_{-}(k)+\mathrm{e}^{3 k / 2} \widehat{q}_{3}(k)-\mathrm{e}^{k / 2} \widehat{\phi}_{+}(k)-\mathrm{e}^{k / 2} \widehat{X}_{1}^{(1)}(k), \\
& \widehat{b}_{1}^{(2)}(k)=\mathrm{e}^{k} \widehat{\phi}_{-}(k)-\mathrm{e}^{-k / 2} \widehat{X}_{2}^{(2)}(k), \\
& \widehat{b}_{2}^{(2)}(k)=\mathrm{e}^{k} \widehat{\phi}_{-}(k)+\mathrm{e}^{k} \widehat{q}_{2}(k)-\widehat{X}_{1}^{(1)}(k)-\mathrm{e}^{-k / 2} \widehat{X}_{2}^{(3)}(k), \\
& \widehat{b}_{3}^{(2)}(k)=\mathrm{e}^{k} \widehat{\phi}_{-}(k)+\mathrm{e}^{k} \widehat{q}_{3}(k)-\widehat{q}_{2}^{(h)}(k)-\widehat{\Lambda}^{(1)}(k), \\
& \widehat{b}_{4}^{(2)}(k)=\mathrm{e}^{k} \widehat{\phi}_{-}(k)+\mathrm{e}^{2 k} \widehat{q}_{1}(k)-\widehat{q}_{2}^{(h)}(k)-\widehat{\Lambda}^{(3)}(k), \\
& \widehat{b}_{5}^{(2)}(k)=\mathrm{e}^{k} \widehat{\phi}_{-}(k)+\mathrm{e}^{2 k} \widehat{q}_{1}(k)+\mathrm{e}^{k} \widehat{q}_{3}(k)-\mathrm{e}^{k} \widehat{q}_{2}(k)-\widehat{X}_{2}^{(1)}(k)-\mathrm{e}^{k / 2} \widehat{X}_{1}^{(3)}(k), \\
& \widehat{b}_{6}^{(2)}(k)=\mathrm{e}^{k} \widehat{\phi}_{-}(k)+\mathrm{e}^{2 k} \widehat{q}_{2}(k)-\mathrm{e}^{k / 2} \widehat{X}_{1}^{(2)}(k), \\
& \widehat{b}_{1}^{(3)}(k)=\mathrm{e}^{3 k / 2} \widehat{\phi}_{-}(k)-\widehat{X}_{1}^{(3)}(k), \\
& \widehat{b}_{2}^{(3)}(k)=\mathrm{e}^{3 k / 2} \widehat{\phi}_{-}(k)+\mathrm{e}^{k / 2} \widehat{q}_{3}(k)-\widehat{X}_{1}^{(2)}(k), \\
& \widehat{b}_{3}^{(3)}(k)=\mathrm{e}^{3 k / 2} \widehat{\phi}_{-}(k)+\mathrm{e}^{3 k / 2} \widehat{q}_{2}(k)+\mathrm{e}^{-k / 2} \widehat{X}_{2}^{(1)}(k) \\
& \quad-\mathrm{e}^{3 k / 2} \widehat{q}_{1}(k)-\mathrm{e}^{-k / 2} \widehat{q}_{3}^{(h)}(k)-\widehat{\Lambda}^{(2)}(k), \\
& \widehat{b}_{4}^{3 k / 2} \widehat{\phi}_{-}(k)+\mathrm{e}^{5 k / 2} \widehat{q}_{1}(k)-\mathrm{e}^{5 k / 2} \widehat{\phi}_{-}(k)-\widehat{X}_{2}^{(3)}(k) .
\end{aligned}
$$

For the uppercase functions $B_{j}^{(n)}(x)$ we find

$$
\begin{aligned}
& \widehat{B}_{1}^{(1)}(k)=\mathrm{e}^{-k / 2} \widehat{\Lambda}^{(1)}(k)-\mathrm{e}^{-k / 2} \widehat{X}_{2}^{(1)}(k), \\
& \widehat{B}_{2}^{(1)}(k)=\mathrm{e}^{-k / 2} \widehat{X}_{2}^{(1)}(k)+\widehat{X}_{2}^{(2)}(k)-\mathrm{e}^{-k / 2} \widehat{q}_{3}^{(h)}(k)-\widehat{\Lambda}^{(2)}(k), \\
& \widehat{B}_{3}^{(1)}(k)=\mathrm{e}^{-k / 2} \widehat{q}_{3}^{(h)}(k)+\mathrm{e}^{k / 2} \widehat{X}_{1}^{(1)}(k)-\mathrm{e}^{k / 2} \widehat{q}_{3}(k)-\widehat{X}_{2}^{(2)}(k), \\
& \widehat{B}_{4}^{(1)}(k)=\mathrm{e}^{k / 2} \widehat{q}_{3}(k)+\mathrm{e}^{k / 2} \widehat{\Lambda}^{(1)}(k)-\mathrm{e}^{k / 2} \widehat{\phi}_{+}(k)-\mathrm{e}^{k / 2} \widehat{X}_{1}^{(1)}(k), \\
& \widehat{B}_{1}^{(2)}(k)=\mathrm{e}^{-k / 2} \widehat{\Lambda}^{(2)}(k)-\mathrm{e}^{-k / 2} \widehat{X}_{2}^{(2)}(k), \\
& \widehat{B}_{2}^{(2)}(k)=\widehat{q}_{2}^{(h)}(k)+\mathrm{e}^{-k / 2} \widehat{X}_{2}^{(2)}(k)-\widehat{X}_{1}^{(1)}(k)-\mathrm{e}^{-k / 2} \widehat{X}_{2}^{(3)}(k), \\
& \widehat{B}_{3}^{(2)}(k)=\widehat{X}_{1}^{(1)}(k)+\widehat{X}_{2}^{(1)}(k)-\widehat{q}_{2}^{(h)}(k)-\widehat{\Lambda}^{(1)}(k), \\
& \widehat{B}_{4}^{(2)}(k)=\mathrm{e}^{k / 2} \widehat{X}_{1}^{(3)}(k)+\mathrm{e}^{-k / 2} \widehat{X}_{2}^{(3)}(k)-\widehat{q}_{2}^{(h)}(k)-\widehat{\Lambda}^{(3)}(k), \\
& \widehat{B}_{5}^{(2)}(k)=\widehat{q}_{2}^{(h)}(k)+\mathrm{e}^{k / 2} \widehat{X}_{1}^{(2)}(k)-\mathrm{e}^{k} \widehat{q}_{2}(k)-\widehat{X}_{2}^{(1)}(k)-\mathrm{e}^{k / 2} \widehat{X}_{1}^{(3)}(k), \\
& \widehat{B}_{6}^{(2)}(k)=\mathrm{e}^{k} \widehat{q}_{2}(k)+\mathrm{e}^{k / 2} \widehat{\Lambda}^{(2)}(k)-\mathrm{e}^{k / 2} \widehat{X}_{1}^{(2)}(k), \\
& \widehat{B}_{1}^{(3)}(k)=\mathrm{e}^{-k / 2} \widehat{\Lambda}^{(3)}(k)-\widehat{X}_{1}^{(3)}(k), \\
& \widehat{B}_{2}^{(3)}(k)=\mathrm{e}^{-k / 2} \widehat{q}_{3}^{(h)}(k)+\widehat{X}_{1}^{(3)}(k)-\widehat{X}_{1}^{(2)}(k),
\end{aligned}
$$




$$
\begin{aligned}
& \widehat{B}_{3}^{(3)}(k)=\widehat{X}_{1}^{(2)}(k)+\widehat{X}_{2}^{(3)}(k)-\mathrm{e}^{3 k / 2} \widehat{q}_{1}(k)-\mathrm{e}^{-k / 2} \widehat{q}_{3}^{(h)}(k)-\widehat{\Lambda}^{(2)}(k), \\
& \widehat{B}_{4}^{(3)}(k)=\mathrm{e}^{3 k / 2} \widehat{q}_{1}(k)+\mathrm{e}^{k / 2} \widehat{\Lambda}^{(3)}(k)-\mathrm{e}^{5 k / 2} \widehat{\phi}_{-}(k)-\widehat{X}_{2}^{(3)}(k) .
\end{aligned}
$$

The crucial point is the observation that the latter forms a system of 14 linear equations, exactly as many as there are unknown functions besides $\widehat{B}_{j}^{(n)}(k)$. Therefore, we can solve (76) to get $\widehat{q}_{1}(k), \widehat{q}_{2}(k), \widehat{q}_{3}(k), \widehat{\Lambda}^{(1)}(k), \widehat{\Lambda}^{(2)}(k), \widehat{\Lambda}^{(3)}(k), \widehat{q}_{2}^{(h)}(k), \widehat{q}_{3}^{(h)}(k)$, $\widehat{X}_{1}^{(1)}(k), \widehat{X}_{2}^{(1)}(k), \widehat{X}_{1}^{(2)}(k), \widehat{X}_{2}^{(2)}(k), \widehat{X}_{1}^{(3)}(k)$ and $\widehat{X}_{2}^{(3)}(k)$ in terms of the auxiliary functions $\widehat{B}_{j}^{(n)}(k)$. Eventually, we substitute this result into (75) and are left with a set of 14 equations, in which only $\widehat{b}_{j}^{(n)}(k)$ and $\widehat{B}_{j}^{(n)}(k)$ appear.

We combine the results from the cases $k<0$ and $k>0$ to get a system of equations valid for all $k \in \mathbb{R}$. We find the equations to be

$$
\widehat{\mathbf{b}}(k)=-\mathrm{i} N \sinh (k \beta / N) \widehat{\mathbf{V}}(k)+\underline{\widehat{\mathbf{K}}}(k) \cdot \widehat{\mathbf{B}}(k),
$$

where

$$
\begin{aligned}
\widehat{\mathbf{b}} & =\left(\widehat{b}_{1}^{(1)}, \ldots, \widehat{b}_{4}^{(1)}, \widehat{b}_{1}^{(2)}, \ldots, \widehat{b}_{6}^{(2)}, \widehat{b}_{1}^{(3)}, \ldots, \widehat{b}_{4}^{(3)}\right)^{\mathrm{T}}, \\
\widehat{\mathbf{B}} & =\left(\widehat{B}_{1}^{(1)}, \ldots, \widehat{B}_{4}^{(1)}, \widehat{B}_{1}^{(2)}, \ldots, \widehat{B}_{6}^{(2)}, \widehat{B}_{1}^{(3)}, \ldots, \widehat{B}_{4}^{(3)}\right)^{\mathrm{T}}, \\
\widehat{\boldsymbol{V}} & =\left(\widehat{V}_{[4]}^{(1)}, \ldots, \widehat{V}_{[4]}^{(1)}, \widehat{V}_{[4]}^{(2)}, \ldots, \widehat{V}_{[4]}^{(2)}, \widehat{V}_{[4]}^{(3)}, \ldots, \widehat{V}_{[4]}^{(3)}\right)^{\mathrm{T}} .
\end{aligned}
$$

The matrix $\underline{\widehat{\mathbf{K}}}(k)$ is exactly the Fourier transform of the kernel matrix (31), and the function

$$
\widehat{V}_{[q]}^{(j)}(k)=\frac{\sinh ([q-j] k / 2)}{\sinh (q k / 2)}
$$

is the Fourier transform of (38). Only the first term on the right-hand side of (77) contains the Trotter number $N$ explicitly. To analytically perform the global Trotter limit $N \rightarrow \infty$, we therefore just have to consider

$$
\lim _{N \rightarrow \infty} N \sinh (k \beta / N)=k \beta .
$$

Next, we apply the inverse Fourier transform and an integration over $x$ to equation (77) to eventually obtain the NLIE (26) $-(38)$. The missing integration constants are determined by considering (26) in the limit $x \rightarrow \infty$. The asymptotics of the auxiliary functions can be easily read off from (73) and (74), because only the constant factors on the right-hand sides survive for large $x$. For the convolutions with the kernel functions, we find

$$
\lim _{x \rightarrow \infty}\left[K_{i} * B_{j}^{(n)}\right](x)=B_{j}^{(n)}(\infty) \int_{-\infty}^{\infty} K_{i}(x) \frac{\mathrm{d} x}{2 \pi}=B_{j}^{(n)}(\infty) \widehat{K}_{i}(0) .
$$

Inserting this information into (26) finally leads to the constants given in (39). 
To also derive the formula for the largest eigenvalue (40), one has to recall that the eigenvalue already appeared during the calculation. Its logarithmic Fourier transform $\widehat{\Lambda}^{(1)}(k)$ could be expressed solely in terms of $\widehat{B}_{j}^{(n)}(k)$. We additionally define the function

$$
\underline{\Lambda}^{(1)}(x)=\frac{\Lambda^{(1)}(x)}{\phi_{-}(x-\mathrm{i}) \phi_{+}(x+\mathrm{i})},
$$

which has the advantage of having constant asymptotics. In the Trotter limit $N \rightarrow \infty$, this definition simply yields $\ln \Lambda^{(1)}(0)=\ln \underline{\Lambda}^{(1)}(0)-\beta$. Using the previously obtained information on $\widehat{\Lambda}^{(1)}(k)$, we can write

$$
\underline{\widehat{\Lambda}}^{(1)}(k)=\mathrm{i} N \sinh (k \beta / N) \mathrm{e}^{-|k| / 2} \frac{\sinh (3 k / 2)}{\sinh (2 k)}+\sum_{n=1}^{3} \sum_{j=1}^{d_{n}} \widehat{V}_{[4]}^{(n)}(k) \cdot \ln \widehat{B}_{j}^{(n)}(k) .
$$

Now we proceed as above. To perform the Trotter limit, we just have to use (82). Then we apply the inverse Fourier transform and an integration over the spectral parameter. Again, we find the integration constant by considering the limit $x \rightarrow \infty$. We finally arrive at equation (40).

\section{References}

[1] H. Bethe, Z. Phys. 71, 205 (1931).

[2] G. V. Uimin, JETP Lett. 12, 225 (1970).

[3] B. Sutherland, Phys. Rev. B 12, 3795 (1975).

[4] J. H. H. Perk and C. L. Schultz, Phys. Lett. A 84, 407 (1981).

[5] N. Andrei and H. Johannesson, Phys. Lett. A 104, 370 (1984).

[6] H. Johannesson, Nucl. Phys. B 270, 235 (1986).

[7] I. Affleck, Nucl. Phys. B 265, 409 (1986).

[8] I. Affleck, Nucl. Phys. B 305, 582 (1988).

[9] C. N. Yang and C. P. Yang, J. Math. Phys. 10, 1115 (1969).

[10] C. P. Yang, Phys. Rev. A 2, 154 (1970).

[11] M. Takahashi, Prog. Theor. Phys. 46, 401 (1971).

[12] M. Gaudin, Phys. Rev. Lett. 26, 1301 (1971).

[13] H. Johannesson, Phys. Lett. A 116, 133 (1986).

[14] P. Schlottmann, Phys. Rev. B 45, 5293 (1992). 
[15] K. Lee, J. Korean Phys. Soc. 27, 205 (1994).

[16] K. Lee, Phys. Lett. A 187, 112 (1994).

[17] M. Suzuki, Phys. Rev. B 31, 2957 (1985).

[18] A. Klümper, Ann. Phys. (Leipzig) 1, 540 (1992).

[19] A. N. Kirillov and N. Y. Reshetikhin, J. Phys. A: Math. Gen. 20, 1565 (1987).

[20] V. V. Bazhanov and N. Y. Reshetikhin, J. Phys. A: Math. Gen. 23, 1477 (1990).

[21] A. Klümper and P. A. Pearce, Physica A 183, 304 (1992).

[22] A. Kuniba, T. Nakanishi, and J. Suzuki, Int. J. Mod. Phys. A 9, 5215 (1994).

[23] Z. Tsuboi, J. Phys. A: Math. Gen. 30, 7975 (1997).

[24] Z. Tsuboi, Physica A 252, 565 (1998).

[25] G. Jüttner, A. Klümper, and J. Suzuki, Nucl. Phys. B 512, 581 (1998).

[26] A. Klümper and M. T. Batchelor, J. Phys. A: Math. Gen. 23, L189 (1990).

[27] A. Klümper, M. T. Batchelor, and P. A. Pearce, J. Phys. A: Math. Gen. 24, 3111 (1991).

[28] C. Destri and H. J. de Vega, Phys. Rev. Lett. 69, 2313 (1992).

[29] A. Klümper, Z. Phys. B 91, 507 (1993).

[30] J. Suzuki, J. Phys. A: Math. Gen. 32, 2341 (1999).

[31] G. Jüttner and A. Klümper, Europhys. Lett. 37, 335 (1997).

[32] G. Jüttner, A. Klümper, and J. Suzuki, Nucl. Phys. B 487, 650 (1997).

[33] A. Fujii and A. Klümper, Nucl. Phys. B 546, 751 (1999).

[34] M. Takahashi, in Physics and Combinatorics 2000, edited by A. N. Kirillov and N. Liskova (World Scientific, Singapore, 2001), pp. 299-304.

[35] Z. Tsuboi, J. Phys. A: Math. Gen. 36, 1493 (2003).

[36] M. Shiroishi and M. Takahashi, Phys. Rev. Lett. 89, 117201 (2002).

[37] Z. Tsuboi, Nucl. Phys. B 737, 261 (2006).

[38] Y. Yamashita, N. Shibata, and K. Ueda, Phys. Rev. B 58, 9114 (1998).

[39] Y. Yamashita, N. Shibata, and K. Ueda, Phys. Rev. B 61, 4012 (2000).

[40] B. Frischmuth, F. Mila, and M. Troyer, Phys. Rev. Lett. 82, 835 (1999). 
[41] N. Fukushima, J. Phys. Soc. Jpn. 71, 1238 (2002).

[42] J. Sirker, Phys. Rev. B 69, 104428 (2004).

[43] S.-J. Gu and Y.-Q. Li, Phys. Rev. B 66, 092404 (2002).

[44] Y. Wang, Phys. Rev. B 60, 9236 (1999).

[45] M. T. Batchelor, X.-W. Guan, A. Foerster, and H.-Q. Zhou, New J. Phys. 5, 107 (2003).

[46] M. T. Batchelor, X.-W. Guan, N. Oelkers, K. Sakai, Z. Tsuboi, and A. Foerster, Phys. Rev. Lett. 91, 217202 (2003).

[47] R. J. Baxter, Exactly Solved Models in Statistical Mechanics (Academic Press, London, 1982).

[48] M. Suzuki and M. Inoue, Prog. Theor. Phys. 78, 787 (1987).

[49] J. Suzuki, Y. Akutsu, and M. Wadati, J. Phys. Soc. Jpn. 59, 2667 (1990).

[50] A. Klümper, T. Wehner, and J. Zittartz, J. Phys. A: Math. Gen. 30, 1897 (1997).

[51] J. Suzuki, Phys. Lett. A 195, 190 (1994).

[52] A. Kuniba and J. Suzuki, Commun. Math. Phys. 173, 225 (1995).

[53] A. Kuniba, Y. Ohta, and J. Suzuki, J. Phys. A: Math. Gen. 28, 6211 (1995).

[54] P. P. Kulish and N. Y. Reshetikhin, Sov. Phys. JETP 53, 108 (1981).

[55] S. Eggert, I. Affleck, and M. Takahashi, Phys. Rev. Lett. 73, 332 (1994).

[56] A. Klümper, Euro. Phys. J. B 5, 677 (1998).

[57] S. Lukyanov, Nucl. Phys. B 522, 533 (1998).

[58] A. Klümper and D. C. Johnston, Phys. Rev. Lett. 84, 4701 (2000).

[59] A. Doikou and R. I. Nepomechie, Nucl. Phys. B 521, 547 (1998).

[60] F. H. L. Essler, V. E. Korepin, and K. Schoutens, Phys. Rev. Lett. 68, 2960 (1992). 